\title{
Sleep problems during the menopausal transition: prevalence, impact, and management challenges
}

This article was published in the following Dove Press journal:

Nature and Science of Sleep

\author{
Fiona C Baker ${ }^{1,2}$ \\ Massimiliano de Zambotti' \\ Ian M Colrain ${ }^{1,3}$ \\ Bei Bei ${ }^{4,5}$ \\ 'Center for Health Sciences, SRI \\ International, Menlo Park, CA, USA; \\ ${ }^{2}$ Brain Function Research Group, \\ University of the Witwatersrand, \\ Johannesburg, South Africa; \\ ${ }^{3}$ Melbourne School of Psychological \\ Sciences, University of Melbourne, \\ ${ }^{4}$ Monash Institute of Cognitive and \\ Clinical Neurosciences, School \\ of Psychological Sciences, Faculty \\ of Medicine, Nursing and Health \\ Sciences, Monash University, \\ ${ }^{5}$ Centre for Women's Mental Health, \\ Department of Psychiatry, University \\ of Melbourne, Royal Women's \\ Hospital, Melbourne, VIC, Australia
}

Corresponding author: Fiona C Baker Center for Health Sciences, SRI International, 333 Ravenswood Avenue, Menlo Park, CA 94025, USA

$\mathrm{Tel}+\mathrm{l} 6508593062$

Fax +I 6508592743

Email fiona.baker@sri.com

\begin{abstract}
A substantial number of women experience sleep difficulties in the approach to menopause and beyond, with $26 \%$ experiencing severe symptoms that impact daytime functioning, qualifying them for a diagnosis of insomnia. Here, we review both self-report and polysomnographic evidence for sleep difficulties in the context of the menopausal transition, considering severity of sleep complaints and links between hot flashes (HFs) and depression with poor sleep. Longitudinal population-based studies show that sleep difficulties are uniquely linked with menopausal stage and changes in follicle-stimulating hormone and estradiol, over and above the effects of age. A major contributor to sleep complaints in the context of the menopausal transition is HFs, and many, although not all, HFs are linked with polysomnographic-defined awakenings, with HF-associated wake time contributing significantly to overall wakefulness after sleep onset. Some sleep complaints may be comorbid with depressive disorders or attributed to sleep-related breathing or movement disorders, which increase in prevalence especially after menopause, and for some women, menopause, age, and environmental/behavioral factors may interact to disrupt sleep. Considering the unique and multifactorial basis for sleep difficulties in women transitioning menopause, we describe clinical assessment approaches and management options, including combination treatments, ranging from cognitive behavioral therapy for insomnia to hormonal and nonhormonal pharmacological options. Emerging studies suggest that the impact of severe insomnia symptoms could extend beyond immediate health care usage and quality of life issues to long-term mental and physical health, if left untreated in midlife women. Appropriate treatment, therefore, has immediate benefit as well as advantages for maintaining optimal health in the postmenopausal years.
\end{abstract}

Keywords: insomnia, midlife women, hormone therapy, estradiol, hot flashes, vasomotor symptoms

\section{Introduction}

Menopause, defined by cessation of menses, marks an important transition in reproductive states in women. The menopausal transition, which begins 4-6 years before cessation of menses (occurring at a median age of 51 years), is associated with fluctuating hormone levels and emergence of physiological and psychological symptoms such as hot flashes (HFs), sleep disturbances, mood changes, and vaginal dryness. Symptoms vary between women in frequency, severity, and duration, persisting for several years after menopause in some women. Sleep disturbances are a major complaint of women transitioning menopause ${ }^{1-5}$ and have a far-reaching impact on the quality of life, mood, productivity, and physical health, especially in women in whom sleep disturbances are severe and associated with functional impairment. In this review, we 
discuss sleep-related issues that arise during the menopausal transition, considering subclinical changes in sleep quality as well as severe sleep disturbances that qualify women for insomnia disorder. We discuss the role of HFs in disrupting sleep, links between depression and sleep disturbance, and the potential impact of chronic sleep disturbance on health in midlife women. Finally, we discuss strategies for managing insomnia in the context of menopause.

\section{Defining the menopausal transition}

The menopausal transition refers to the years leading up to menopause, or final menstrual period, when endocrinological, biological, and clinical features of approaching menopause commence. ${ }^{6}$ The median age of onset of the menopausal transition is 47 years, and the median age at final menstrual period is 51.4 years. $^{7}$ The Stages of Reproductive Aging Workshop $\left(\right.$ STRAW) ${ }^{8}$ (updated in 2011 ${ }^{9}$ ) is considered the gold standard for characterizing reproductive aging, broadly grouping women into three categories (reproductive, menopausal transition, and postmenopause) with further subdivisions defined by bleeding criteria and hormone levels (Figure 1).

The early menopausal transition is marked by a persistent difference of $\geq 7$ days in the length of consecutive cycles. ${ }^{9}$ The late menopausal transition is marked by increased variability in cycle length and the occurrence of amenorrhea of $\geq 60$ days. ${ }^{9}$ Hormonal changes roughly map onto these stages, although changes are nonlinear over time. ${ }^{10}$ In the early menopausal transition, follicle-stimulating hormone (FSH) levels (measured in the early follicular phase of the menstrual cycle) are elevated but variable. The majority $(80 \%)$ of cycles are still ovulatory, ${ }^{11,12}$ and thus, menstrual cycle-related hormone changes are superimposed on menopausal transition changes; within any ovulatory menstrual cycle, follicular phase estradiol (E2) levels tend to be lower, luteal phase E2 levels tend to be higher, and luteal phase progesterone levels are lower than in reproductive-aged women. ${ }^{13}$ The late menopausal transition is characterized by FSH levels $\geq 25$ IU/L. ${ }^{9}$ Early postmenopause (first $1-6$ years after final menses) is characterized by a continued rise in FSH levels and decline in E2 levels particularly over the first 2 years after the final menstrual period, ${ }^{9}$ after which FSH levels gradually stabilize. Perimenopause is a term commonly used, especially before STRAW criteria were developed, to describe the menopausal transition and first-year postmenopause, and lasts a median of 4 years. ${ }^{14}$ HFs (or flushes) - a common symptom of impending menopause - typically emerge in the late reproductive stage or early menopausal transition, peak in the late menopausal transition and first 2 years after

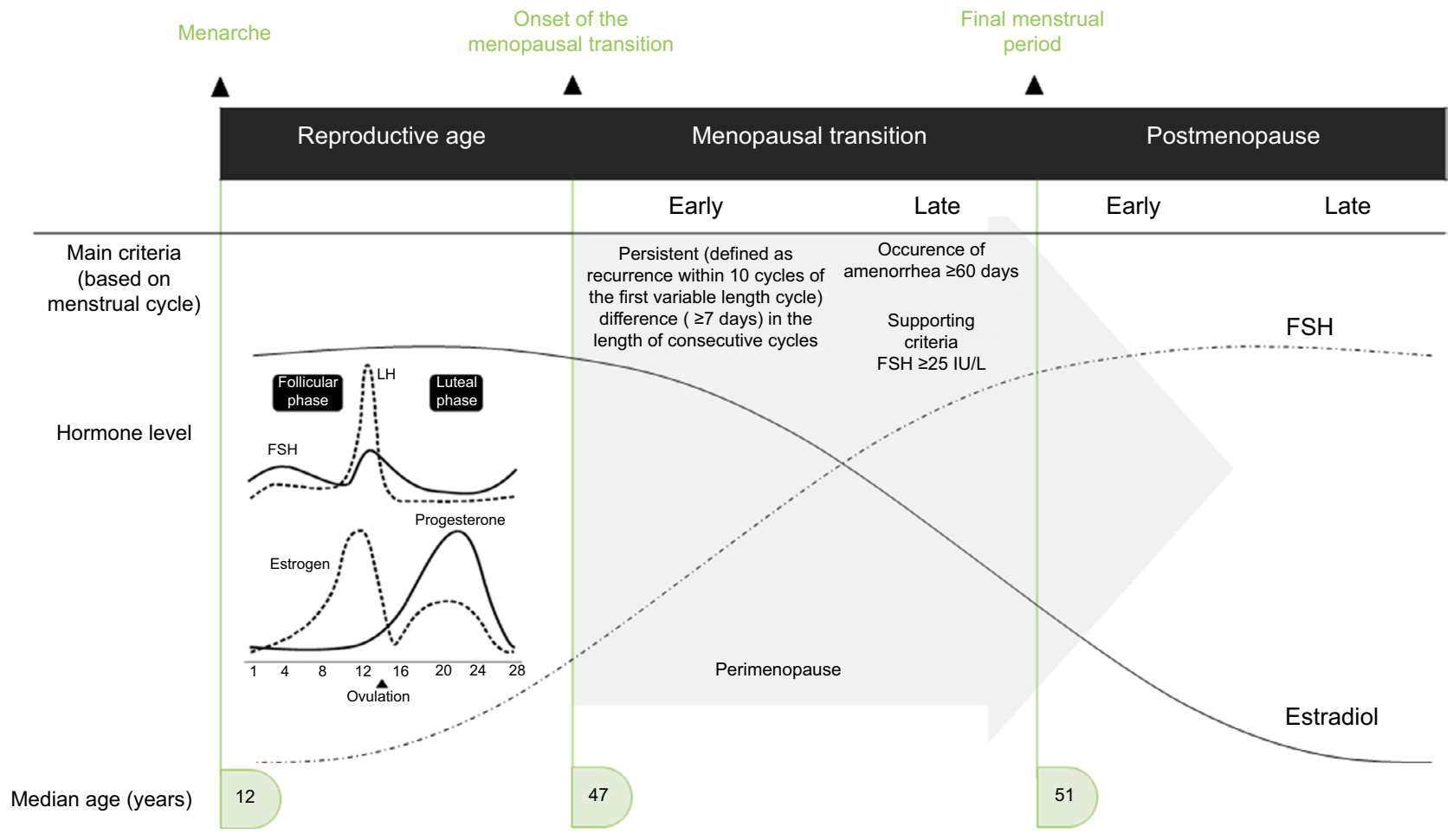

Figure I Progression through the menopausal transition and postmenopause as defined by the Stages of Reproductive Aging Workshop (STRAW).

Notes: Schematic changes in follicle-stimulating hormone and estradiol (follicular phase samples) are superimposed on the STRAW criteria, although there is substantial variability in hormone levels across the transition. Menstrual cycle-related fluctuations in hormones are shown in the insert. Data from Soules et al and Harlow. 89 Abbreviations: FSH, follicle-stimulating hormone; LH, luteinizing hormone; STRAW, Stages of Reproductive Aging Workshop. 
menopause, and decline in late menopause ( $>6$ years after final menstrual period). ${ }^{15}$ Studies have also used the term "vasomotor symptoms" to describe HFs and night sweats.

In women with amenorrhea or unreliable bleeding patterns, such as endometrial ablation, hysterectomy, treatment of malignancy, and chronic illness, endocrine markers of ovarian aging, such as a rise in $\mathrm{FSH}$, are required to identify the timing of the menopausal transition. ${ }^{15}$ Women who have bilateral oophorectomy (removal of ovaries) are plunged into menopause, with a rise in FSH and drop in estradiol, and sudden appearance of menopausal symptoms, which are typically more severe than with natural menopause. ${ }^{16}$ Women with hysterectomy alone (ovaries are intact) and hysterectomy plus oophorectomy may be combined into a single category, "surgical menopause", although it should be kept in mind that the endocrine environment differs between these two groups.

\section{Sleep difficulties reported across the menopausal transition}

Midlife women transitioning menopause and postmenopause are more likely to report sleep difficulties, with prevalence rates of self-report sleep difficulties ranging between $40 \%$ and $56 \%$, compared to premenopausal women in the latereproductive stage, who have rates of $31 \% .{ }^{17}$ This greater prevalence of sleep difficulties in association with the menopausal transition, even after controlling for age, is evident across many cross-sectional studies from different countries despite wide variability in the assessment and definition of sleep difficulties and menopausal staging, and the number of covariates controlled, as shown in a recent meta-analysis of cross-sectional data from 24 studies. ${ }^{18}$ Women in perimenopause (1.60), postmenopause (1.67), and surgical menopause (2.17) had higher odds of experiencing sleep disturbance, relative to premenopause. ${ }^{18}$

Longitudinal studies are well suited to examine the change over time in sleep difficulties as women progress through the menopausal stages as well as in relation to hormone changes. Such studies have mostly confirmed crosssectional data and revealed some nuances in the relationship between menopause and sleep difficulties. ${ }^{19-25}$ Longitudinal analysis of 8 years of data from $>3000$ women in the Study of Women's Health Across the Nation (SWAN) showed that sleep difficulties due to frequent awakenings (over the past 2 weeks), in particular, increased as women transitioned menopause and was the most common problem (Figure 2). ${ }^{22}$ Some differences related to ethnicity emerged, with Caucasian women showing the greatest odds and Hispanic women showing the lowest odds of frequent awakenings, although ethnicity-menopausal stage interaction effects were negligible. ${ }^{22}$ In their longitudinal analysis, Tom et $\mathrm{al}^{24}$ showed the importance of considering severity of sleep difficulties, with a stronger relationship between menopausal stage and bothersome trouble sleeping (over the past year) for those women with severe vs moderate sleep difficulties. Both of these studies confirmed cross-sectional data that women who transitioned to surgical menopause and were not taking hormone therapy (HT) had the greatest odds of sleep difficulty (Box 1). ${ }^{22,24}$ The Penn Ovarian Aging Study did not observe a relationship between menopausal status or age with sleep

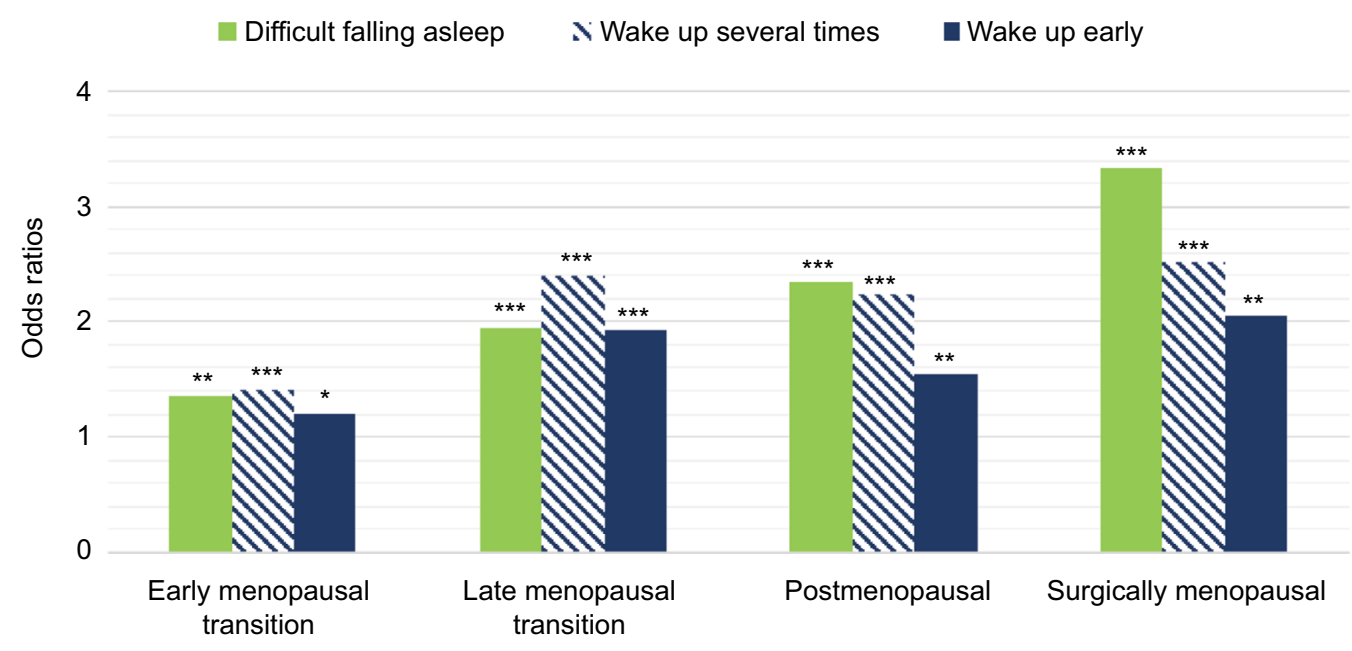

Figure 2 Age-adjusted odds ratios for self-reported sleep difficulties in women participating in the SWAN prospectively tracked across the natural menopausal transition relative to premenopausal baseline and in women who transitioned to surgical menopause.

Notes: $* P<0.05$; ${ }^{* * P} P 0.01$; ${ }^{* * *} P<0.001$. Data from Kravitz et al. ${ }^{22}$

Abbreviation: SWAN, Study of Women's Health Across the Nation. 
Box I The impact of surgical menopause or treatment of malignancy on sleep quality

Surgical menopause is associated with more severe sleep disturbances than natural menopause. ${ }^{18,22,24,26} \mathrm{Kravitz}$ et al ${ }^{17}$ reported that women in the SWAN study who had a bilateral oophorectomy and who were not using HT had the highest prevalence of sleep difficulty, independent of age or years since surgery, with this effect related to HFs. Indeed, women who undergo bilateral oophorectomy are at increased risk for more severe HFs than women in natural menopause, ${ }^{16}$ which likely impact sleep. An additional possible reason for greater sleep difficulties in women with surgical menopause (whether or not including bilateral oophorectomy) relative to natural menopause could be an underlying worse health profile prior to menopause or worse health after surgery. ${ }^{24}$ Use of $\mathrm{HT}$ effectively improves sleep in surgically menopausal women.

Women undergoing treatment for malignancy frequently experience symptoms of menopause. In premenopausal women prescribed tamoxifen, which blocks the effects of estrogen on cancer cells, $\mathrm{FSH}$ and estradiol levels are altered ${ }^{15}$ and $80 \%$ of them develop HFs, ${ }^{27}$ which are more likely to be severe compared with other breast cancer survivors. ${ }^{28}$ Tamoxifen use is also associated with sleep difficulties, ${ }^{28}$ likely linked with HFs since $\mathrm{HFs}$ are specifically associated with poor sleep ${ }^{29,30}$ and polysomnographic-defined sleep disturbance ${ }^{31}$ in breast cancer survivors. Since antiestrogen therapy such as tamoxifen may be administered for at least 5 years, or even extended up to 10 years, symptoms can be present for a long time and effective supportive management is required. ${ }^{27} \mathrm{HT}$ is contraindicated in women with, or at high risk for, breast cancer, and therefore, other options - taking into account symptom severity - should be considered, including pharmacological (eg, antidepressants) and nonpharmacological (eg, behavioral modifications and cognitive behavioral therapy) approaches. ${ }^{27}$

Abbreviations: SWAN, Study of Women's Health Across the Nation; HT, hormone therapy; HFs, hot flashes; FSH, follicle-stimulating hormone.

quality assessed for previous night $\mathrm{t}^{23}$ or any difference in the prevalence of "trouble sleeping over the past month" relative to the final menstrual period in the sample overall, ${ }^{21}$ although there was a strong association between vasomotor symptoms and poor sleep regardless of stage. ${ }^{21,23}$ An important finding from this study was that premenopausal sleep status was the strongest predictor of poor sleep around the final menstrual period, supporting other work that a history of insomnia is a strong predictor of current symptoms. ${ }^{32}$

Some studies have considered relationships between hormone levels (as opposed to only bleeding patterns) and sleep difficulties. In the Seattle Midlife Women's Health Study cohort, higher FSH and lower estrone levels were associated with greater severity of nighttime awakenings; however, this relationship did not remain significant in multivariate analysis. ${ }^{25}$ In a cross-sectional study of 160 peri- and postmenopausal women, no relationship was found between E2 or FSH with diary-reported sleep measures, although wake time was strongly associated with menopausal stage. ${ }^{33}$ Longitudinal studies are better suited for tracking changes in hormone levels in relation to changes in sleep: SWAN found that decreasing E2 and increasing FSH levels were associated with higher odds of frequent awakenings across follow-ups ${ }^{22}$ and that a greater rate of change in FSH was associated with poorer sleep quality. ${ }^{34} \mathrm{Also}$, the 13 -year prospective Melbourne Women's Midlife Health Project found that a steeper E2 slope was associated with more severe sleep problems, ${ }^{10}$ and some evidence suggests that declining estradiol levels are associated with worsening sleep quality even in late reproductive stage women. ${ }^{35}$

Together with evidence of worsening sleep quality following oophorectomy, when the change in hormones is sudden, these results imply that a faster change in hormones is associated with a poorer sleep profile. In summary, there is an increase in the prevalence of sleep difficulties as women traverse menopause, evident in relation to menopause stages based on bleeding patterns as well as endocrine changes. Sleep problems are multifactorial, strongly associated with HFs and depression (as discussed later), as well as other factors coincident with aging into midlife including stress, poor health and chronic pain. ${ }^{25,36,37}$ There is also an agerelated increase in the presence of sleep-related breathing and movement disorders, as discussed later. These factors may also interact with each other, providing a complex basis for sleep difficulties in midlife women. As such, investigations of subgroups of women ${ }^{38}$ and consideration of clusters of symptoms in women rather than just focusing on the menopausal transition ${ }^{25}$ may be valuable approaches. Limited data also suggest that differences in circadian regulation (advanced circadian phase) could contribute to sleep difficulties, ${ }^{39}$ particularly a more fragmented sleep or early morning awakening, in postmenopausal women. Finally, an additional source of variability to hormone levels and sleep quality when women are in the early menopausal transition is menstrual cycle phase; women have a poorer sleep quality in the late-luteal and early-follicular phases than at other times. $^{40,41}$

\section{Polysomnographic measures in women in the menopausal transition}

Epidemiological studies clearly show a rise in self-reported sleep difficulties as women transition menopause. These data reflect how women are feeling, which is highly relevant to their use of health care and quality of life. ${ }^{22}$ Polysomnography (PSG), including electroencephalographic (EEG) analy- 
sis, provides detailed information about biological sleep and its regulation, and is also a powerful tool for examining relationships between aspects of sleep and other biological measures, such as hormone levels and cardiovascular and respiratory functions. Importantly, while not required for an insomnia diagnosis, PSG (or a reduced subset of these signals) is essential for diagnosing sleep disorders such as obstructive sleep apnea syndrome. However, PSG-derived measures of sleep do not necessarily reflect self-report sleep quality ratings. ${ }^{42}$

Given the multiple factors evident from epidemiological studies that can influence sleep difficulties and their measurement across the menopausal transition (eg, presence of preexisting sleep complaints, severity of sleep complaints, symptoms such as HFs, variable hormone fluctuations, and the influence of other conditions coincident with midlife), it perhaps is not surprising that PSG studies have not consistently shown evidence of sleep disturbances linked directly with menopausal stages (Table 1). Most PSG studies have been observational and conducted in smaller, select samples, on one or only a few nights, which capture a snapshot of sleep. Furthermore, most studies did not control for confounding factors such as age, health status, medications, and presence of a sleep disorder. Two large cohort studies designed to address some of these limitations are the Wisconsin Sleep Cohort and SWAN studies. The Wisconsin Sleep Cohort Study of 589 midlife women showed that peri- and postmenopausal women had better PSG sleep, with more slow wave sleep (SWS), than premenopausal women, ${ }^{43}$ whereas SWAN found no differences in PSG measures according to menopausal status. ${ }^{44}$ However, late-perimenopausal stage and postmenopausal women had more high-frequency beta EEG activity, suggesting greater cortical hyperarousal, during sleep than premenopausal and early-perimenopausal stage women, an effect partially explained by higher frequency of self-report HFs. ${ }^{44}$ Taken together, studies have shown few consistent effects of menopausal stage per se on sleep architecture. Results of SWAN, however, show the potential relevance of considering more complex measures of sleep quality, such as quantitative EEG. Also, taken together, results suggest that while not all women experience sleep disruption in association with the menopausal transition, subgroups might be more susceptible, such as those with severe HFs and/or insomnia disorder. Most importantly, PSG studies have been cross-sectional except for one ${ }^{45}$ and, therefore, are unable to track the trajectories of change in PSG measures in relation to transitioning menopause. Women may begin to experience menopausal symptoms even in the early 40s, when they are still premenopausal, which could confound cross-sectional comparisons between different stages.

Lampio et $\mathrm{al}^{45}$ used a longitudinal design to investigate the changes in PSG measures in 60 midlife women six years after a premenopausal baseline visit. At follow-up, women had a shorter total sleep time, lower sleep efficiency, more wakefulness after sleep onset (WASO), and more awakenings after adjusting for HFs/night sweats, body mass index, and mood, an effect linked with advancing age rather than increased FSH levels. In contrast, increasing FSH was associated with an increased proportion of SWS (although not with slow-wave EEG activity), which the authors propose as reflecting an adaptive change to counteract the age-related sleep fragmentation. ${ }^{45} \mathrm{SWAN}$ data also found a link between the change in FSH over time and more SWS as well as a longer total sleep time, ${ }^{34}$ which contrasts with findings correlating hormone changes with subjective sleep quality as described earlier. The relationship between reproductive hormones and sleep appears to differ in the reproductive years and earlier in the menopausal transition: in a cross-sectional study of young premenopausal and early menopausal transition women, all in the follicular phase, we found a correlational relationship between higher FSH levels and more WASO but not with other sleep measures, as assessed with single-night, in-laboratory $\mathrm{PSG},{ }^{46}$ suggesting an interaction between hormones of the hypothalamic pituitary ovarian axis and sleep-wake regulatory systems that is apparent even in reproductive-age women. These data are correlational, however, and further studies tracking frequent changes in hormone levels in association with sleep are needed. There is growing evidence, from animal models, of the underlying mechanisms of how reproductive hormones act to alter sleep and circadian rhythms, although many knowledge gaps remain and the mechanisms are likely complex. ${ }^{47}$

Interestingly, when we looked separately at women with insomnia disorder developed in the context of the menopausal transition, they did not show any FSH-WASO relationship, possibly due to the influence on their sleep of other factors such as mood, hyperarousal, and night-to-night variability. ${ }^{46}$ These data further indicate the challenge in isolating the influence of hormones from the several other confounding factors that affect sleep.

\section{HFs and reported sleep difficulties}

HFs are a hallmark of the menopausal transition, being reported by up to $80 \%$ of women. ${ }^{48-51} \mathrm{~A} \mathrm{HF}$ is a sensation of 


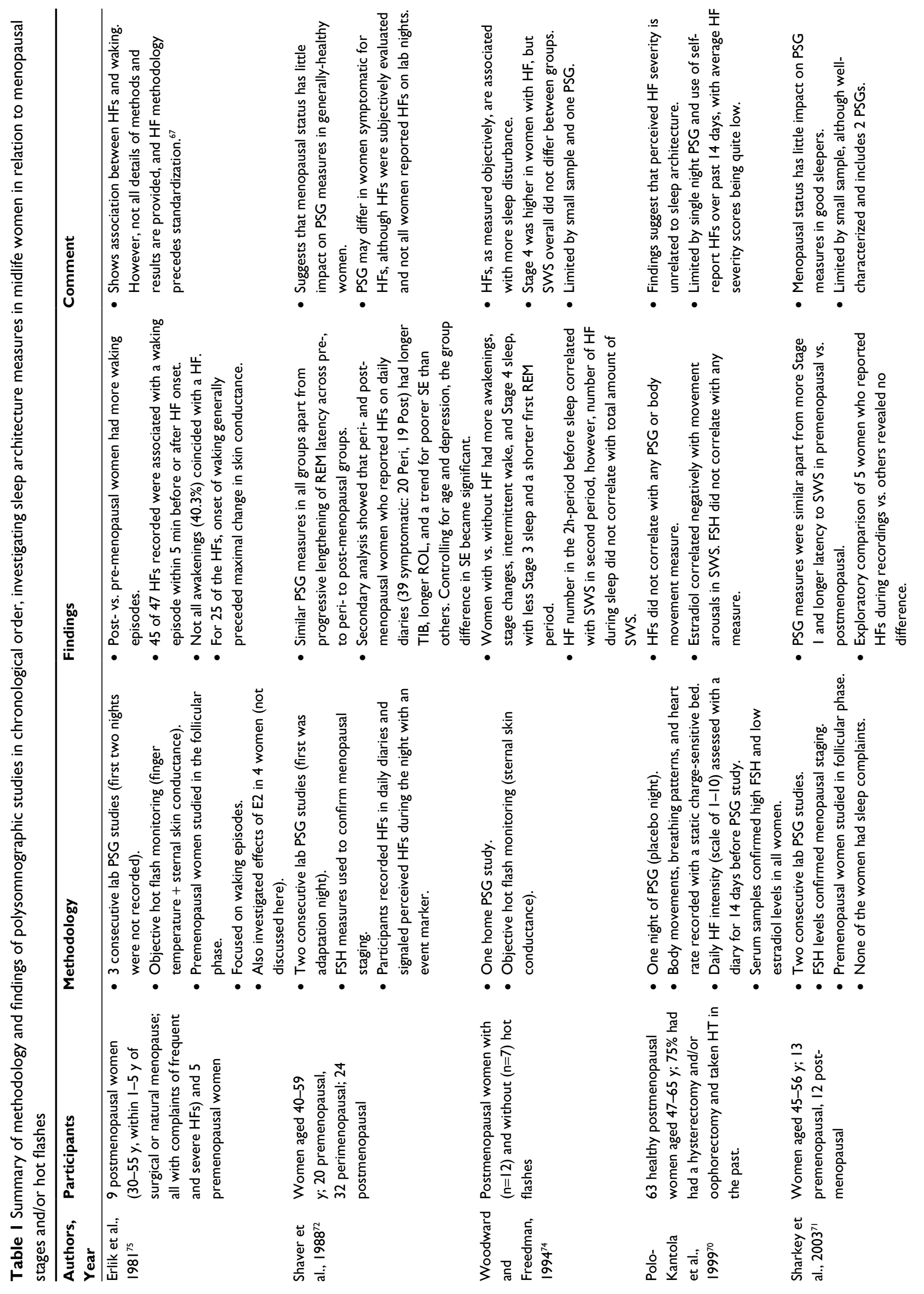



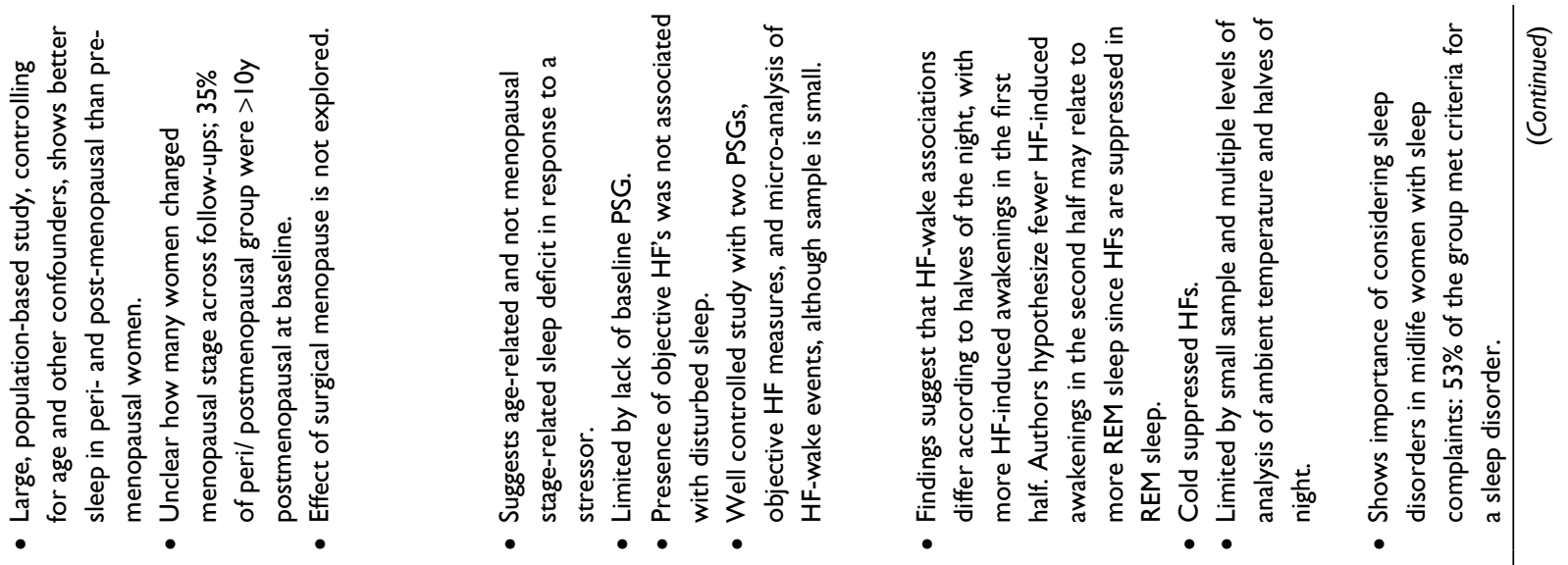

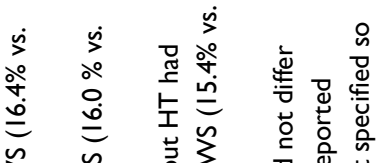

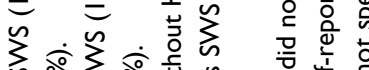

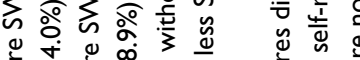

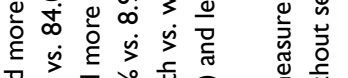

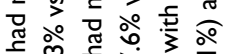

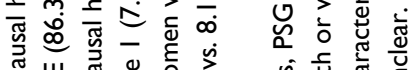

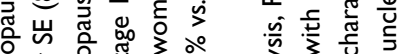

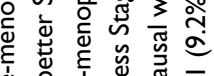

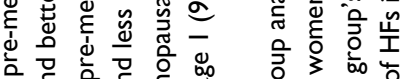

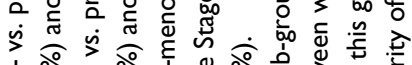

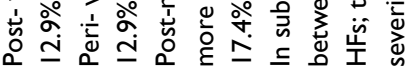

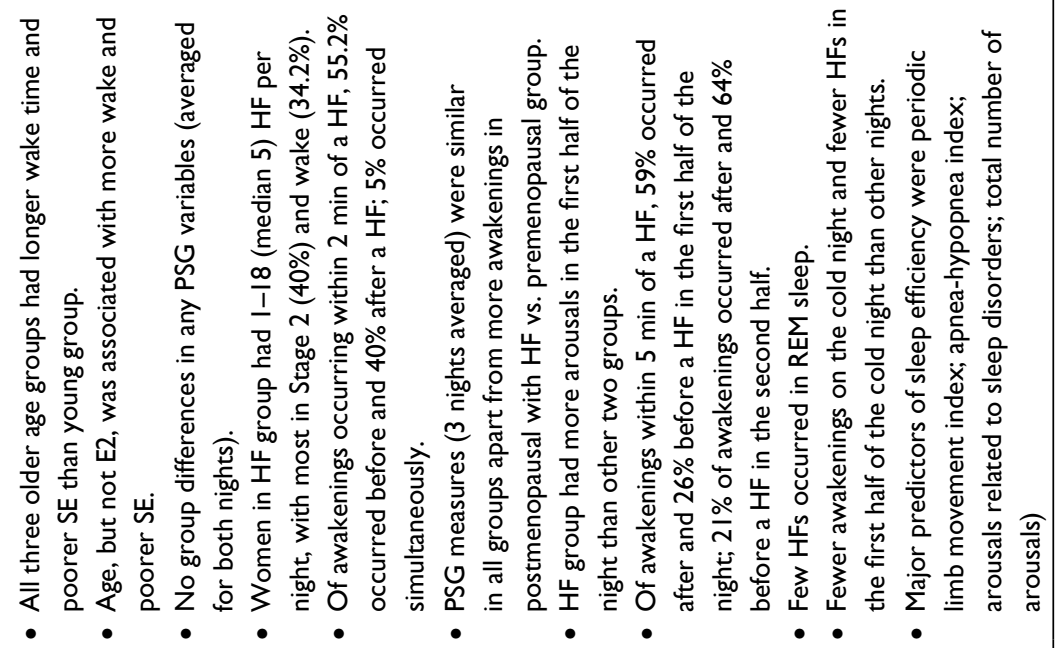

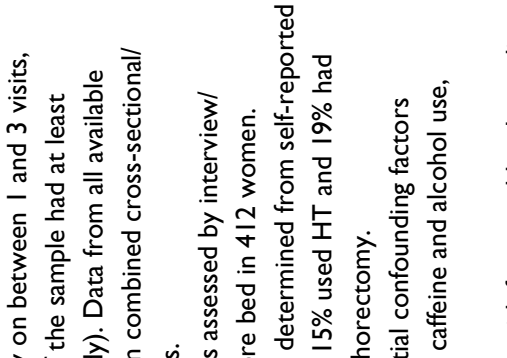

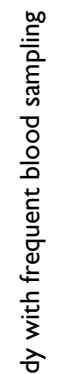

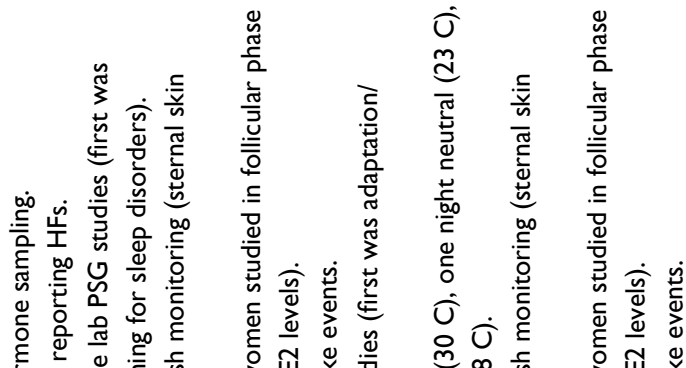

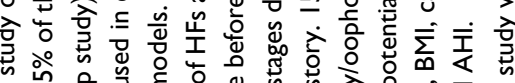

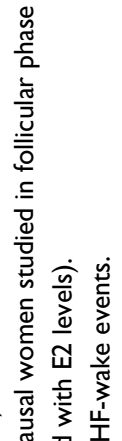

U人

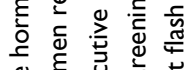

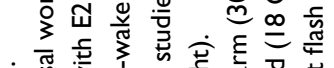

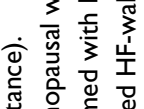

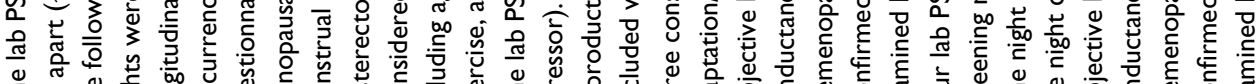

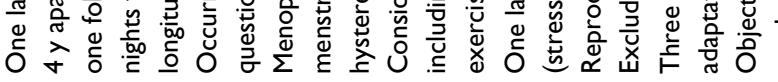

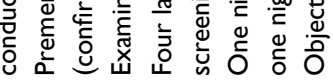

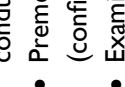

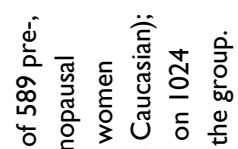

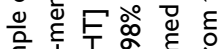

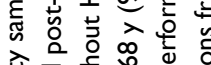

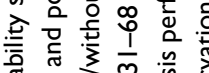

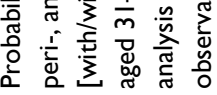

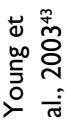
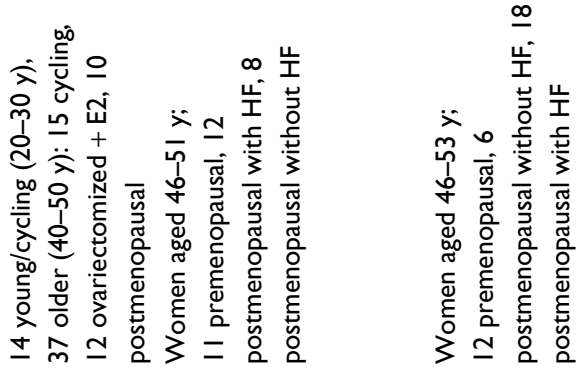

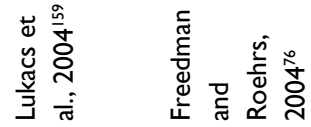

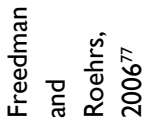

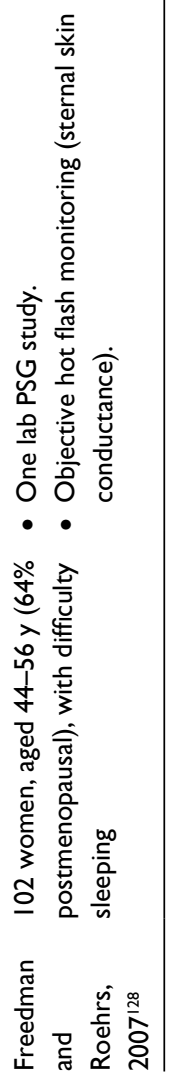




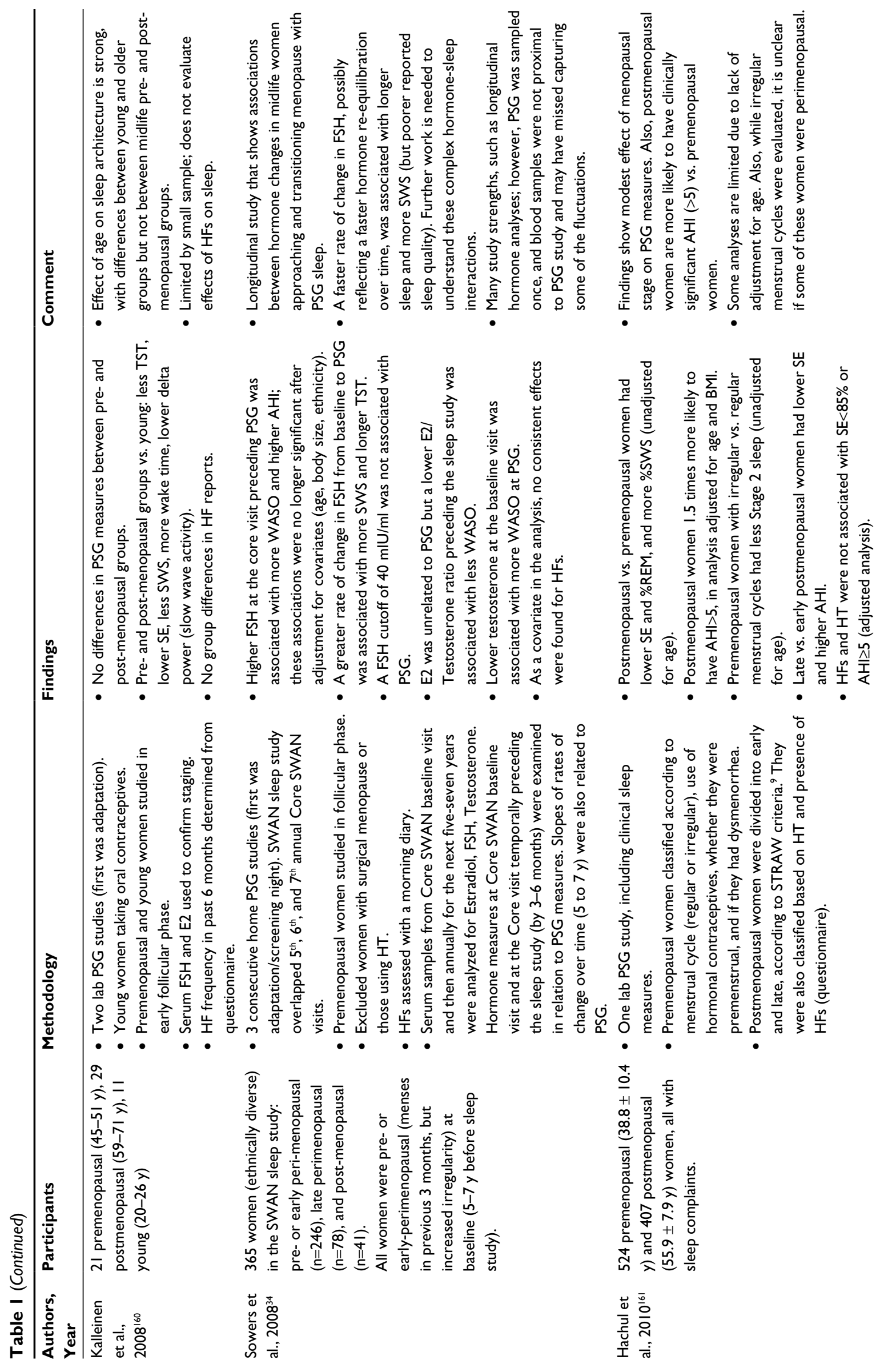



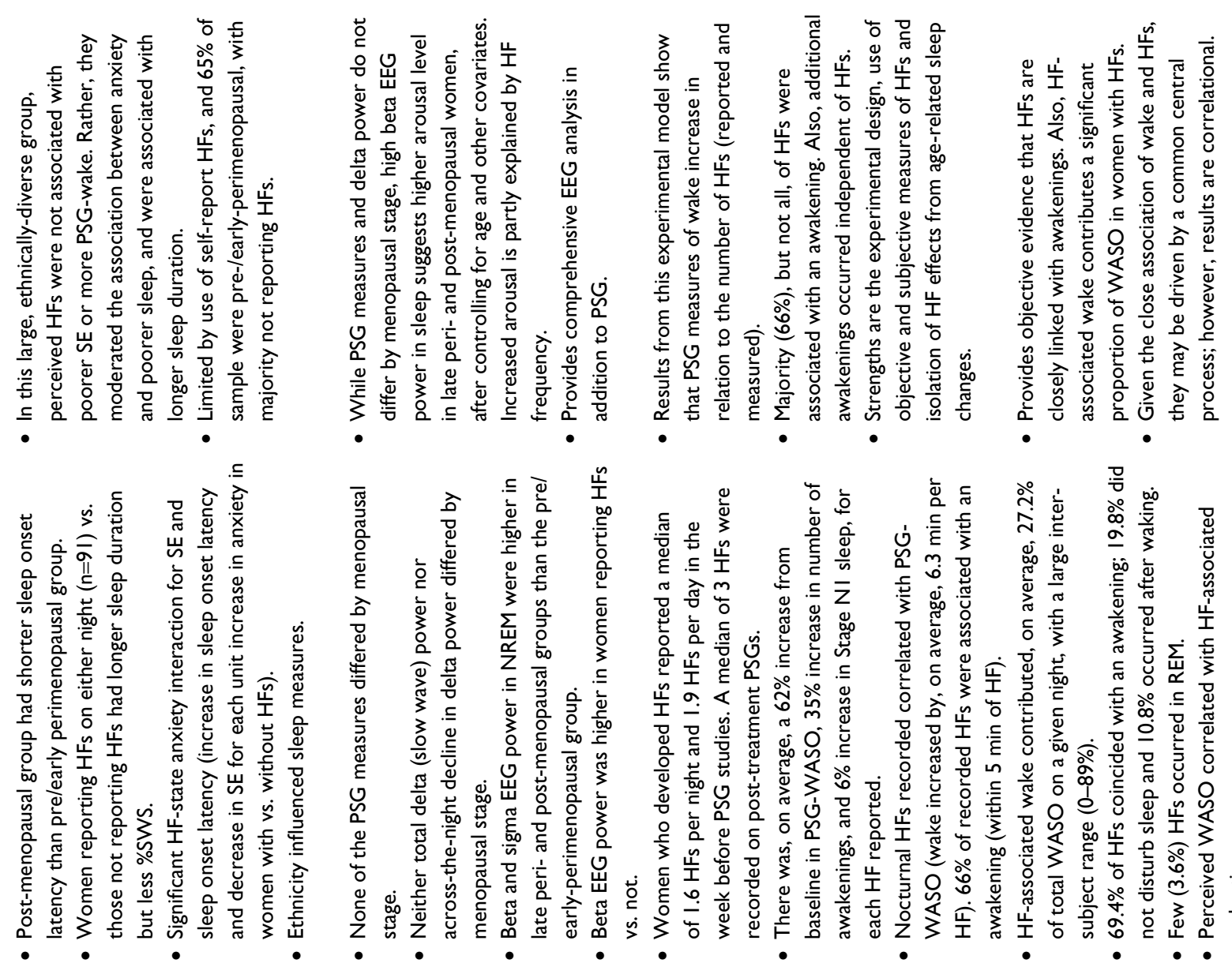

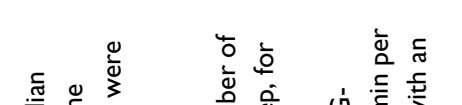

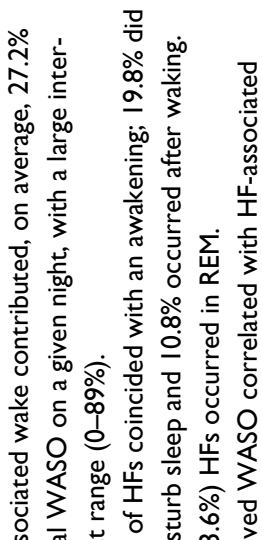
。 d

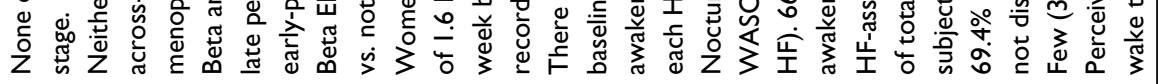
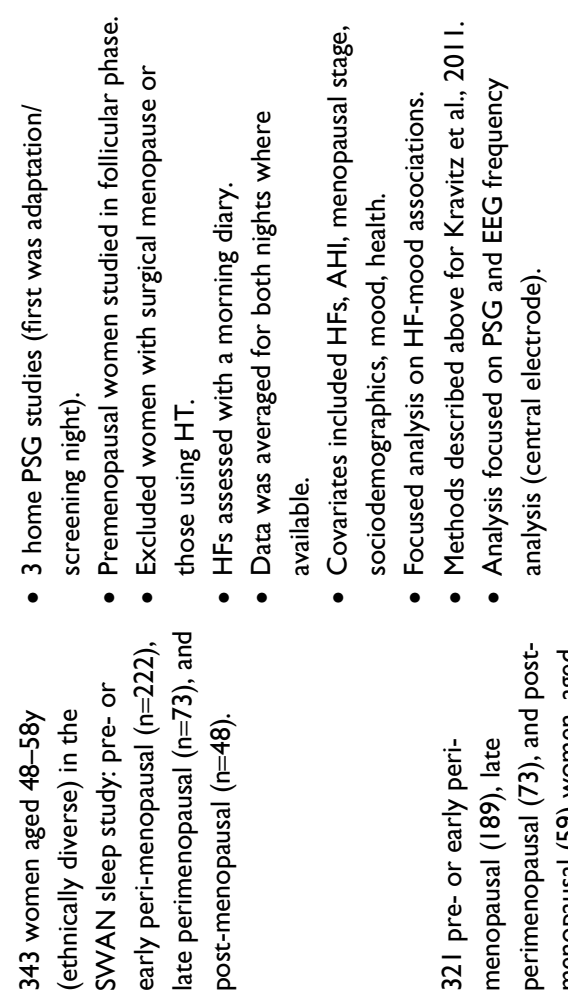

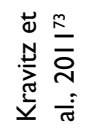

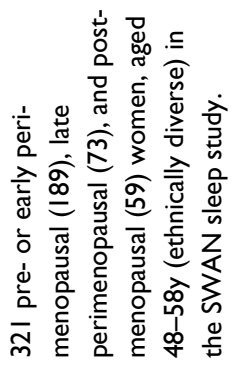

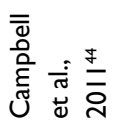
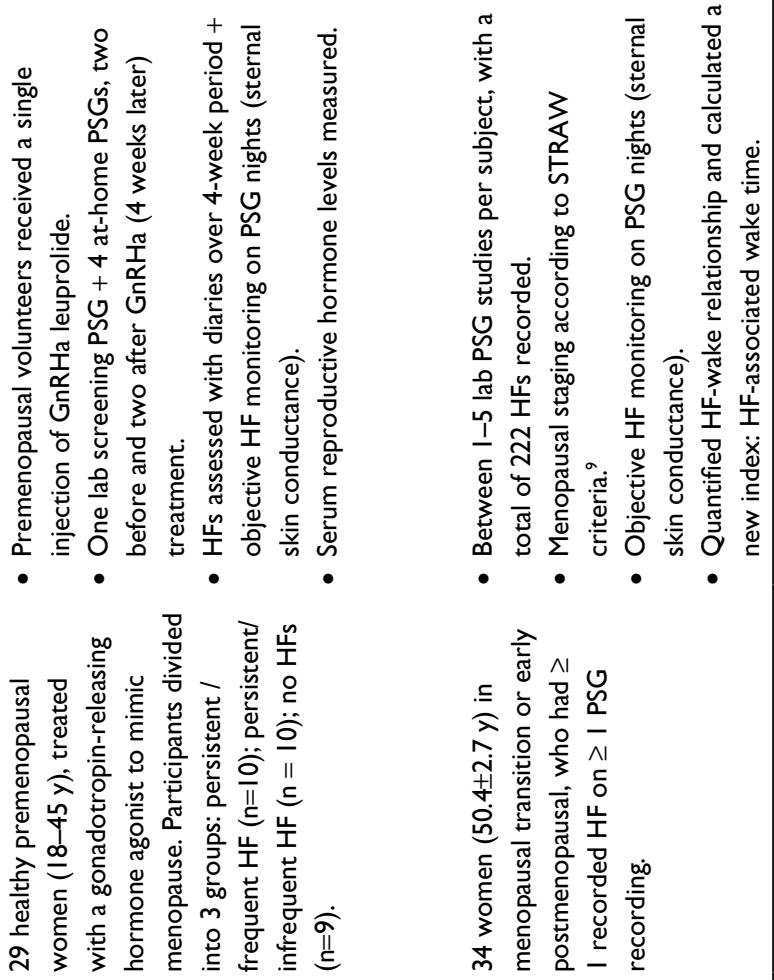

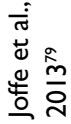

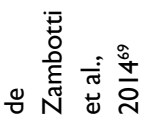




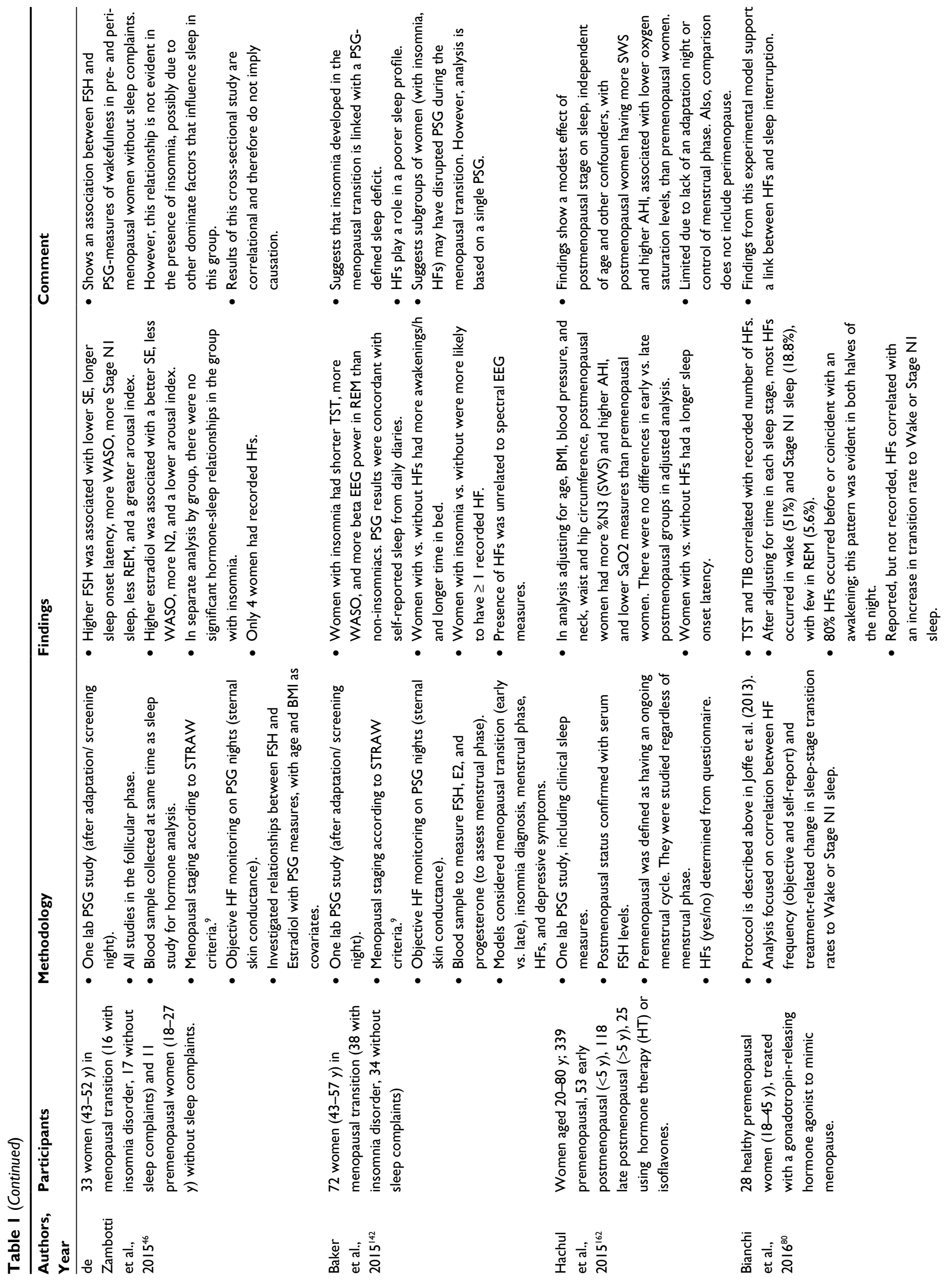



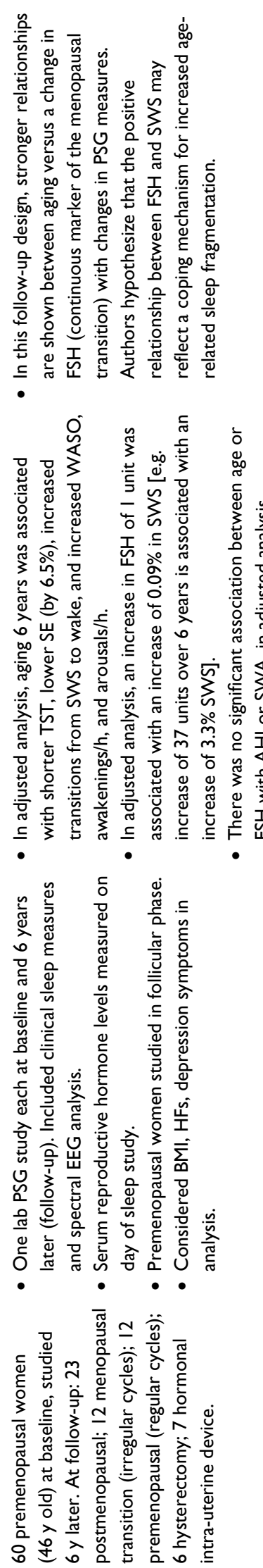

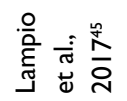

heat, sweating, flashing, anxiety, and chills lasting between 3 and $10 \mathrm{~min}^{52}$ and is primarily a thermoregulatory phenomenon $^{53}$ with characteristics of a heat dissipation response: peripheral vasodilation, which causes increased heat loss, and increased sweating, which causes evaporative cooling. Some women have HFs hourly or daily, and others report only 1 or $2 /$ week. $^{52}$ HFs can occur during the day or night (night sweats). ${ }^{52}$ Data from SWAN show that the median duration of HFs is 7.4 years. ${ }^{48}$ The temporal pattern of HFs across the menopausal transition is not the same for all women, with studies identifying distinct patterns based on the time of symptom onset and persistence. ${ }^{51,54,55}$ The SWAN study showed that these different trajectories are associated with health, with women in the "persistently high/early onset" group having a more adverse health and psychosocial profile. ${ }^{51}$ Also, early-onset HFs are associated with greater subclinical cardiovascular disease. ${ }^{56}$

HFs emerge as estrogen levels decline, but their mechanism is more complex than just estrogen withdrawal, with several lines of evidence implicating the involvement of central noradrenergic activity ${ }^{57-59}$ as well as serotonergic mechanisms, ${ }^{57}$ and most recently, involvement of hypothalamic kisspeptin, neurokinin $\mathrm{B}$, and dynorphin (KNDy) neurons. ${ }^{60}$ Since not all women develop HFs (despite showing the expected decline in estradiol going through menopause) and since severity, frequency, and duration vary among women, neural systems may be altered in vulnerable women, leading them to be more sensitive to the effects of estrogen fluctuations.

The presence of self-reported HFs is consistently associated with poorer self-reported sleep quality and chronic insomnia ${ }^{17,22,37,41,61}$ indicating that women link HFs with nocturnal awakenings. Longitudinal data from SWAN show that women with moderate-to-severe HFs (6-14 days in a 2-week period) are almost three times more likely to report problems of frequent nocturnal awakenings compared to women without HFs. ${ }^{62} \mathrm{HF}$-associated bother and interference over and above frequency appear to be important constructs to consider; $\mathrm{Xu}$ et $\mathrm{a} \mathrm{l}^{63}$ reported that bothersome HF but not HF alone was associated with sleep difficulties. Further supporting HFs as a cause of poor sleep are studies showing that the effective treatment of HFs with HT is associated with improved sleep quality. $2,4,64$ Recently, Pinkerton et $\mathrm{l}^{65}$ analyzed clinical trial data in women with frequent moderate-to-severe HFs ( $\geq 7 /$ day) and quantified the effect on quality of life and sleep problems of reducing the number and severity of HFs with treatment; a reduction of five HFs per day is associated with a clinically meaningful reduction in sleep problems. Critically, HFs can emerge in the late $30 \mathrm{~s}$ 
and 40s, and are associated with poor sleep even at this latereproductive stage. ${ }^{35}$ Given that HFs can continue to persist for several years postmenopause in some women, they may be an important source of sleep disturbance well after midlife. ${ }^{66}$

\section{Polysomnographic measures in women with HFs}

As for PSG studies comparing sleep in women at different menopausal stages, PSG studies comparing sleep in women with and without HFs have produced results inconsistent with self-reported evidence of a strong link between HFs and sleep difficulties (Table 1). Some of these inconsistencies are related to methodological issues of studying sleep in the menopausal transition, as described earlier, and additional methodological challenges associated with HFs. HFs have been assessed based on self-report over a previous averaged time period of days or weeks or during the recording night (with self-report measures differing in the assessment of frequency, severity, and bother), or assessed based on the "objective" measures of sternal skin conductance. An HF is objectively identified by a sudden increase in sternal skin conductivity due to a sweat response, which tails off as sweating decreases, considered the gold standard method of measuring HF frequency. ${ }^{67}$ Concordance between selfreport and objective HFs varies widely between studies and is particularly low due to under reports when an HF occurs at night, ${ }^{67}$ not surprising since retrospective recall of nighttime HF events on the following morning may be inaccurate and confounded by fatigue and $\operatorname{mood}^{64,68}$ as well as being dependent on having woken up at the time of the HF to remember the event. Finally, most studies have been observational rather than using interventions, limited in determining causality between HFs and disturbed sleep. Although self-report HF may be biased by the "retrospective" nature of the morning self-report, it is still clear that women recall the presence of HFs at night only when they are associated with a conscious awakening, and thus, each self-reported HF is truly associated with nocturnal wakefulness, a factor that should increase the strength of the subjective HF-sleep association. In contrast, not all the objectively recorded HFs are associated with awakenings, ${ }^{69}$ a factor that seems to affect the relationship between objective HFs and sleep disruption.

Self-report HFs have been associated with EEG hyperarousal during sleep in the SWAN study, ${ }^{44}$ whereas others showed no differences in PSG markers of sleep disturbance, ${ }^{43,70,71}$ or more time in bed ${ }^{72}$ or longer sleep time ${ }^{73}$ between women reporting HFs vs women not reporting HFs. Similarly, for objectively measured HFs, symptomatic postmenopausal women compared with asymptomatic postmenopausal women had a poorer PSG-defined sleep profile (more awakenings and lower sleep efficiency) in one study ${ }^{74}$ or no difference in PSG measures in another study. ${ }^{76}$ To further explore the relationship between HF and PSG-measured sleep, we and others have conducted more refined analysis on individual HF events and PSG measures in women with HFs in the menopausal transition or postmenopause. ${ }^{69,75-78}$ Studies are consistent in showing a low incidence of HFs in REM sleep ${ }^{69,76,77}$ possibly owing to the inhibition of thermoregulatory responses in this stage. However, studies are not consistent in showing HF-wake associations, with reports of awakenings more likely to occur before an HF than after an $\mathrm{HF}^{76} \mathrm{HFs}$ occurring before an awakening only in the first half of the night, ${ }^{77}$ or HFs coincident with awakenings in the majority of events, ${ }^{69,75,78}$ with no differences in the first and second parts of the night. ${ }^{69}$ We found that $\sim 80 \%$ of HFs in our sample interfered with sleep. ${ }^{69}$ Differences between studies might relate to how HFs were classified as associated and not associated with awakenings and how the windows of analysis were defined, ${ }^{69}$ as well as variability between women in the extent to which HFs disrupt sleep.

Emerging data have shown the importance of using experimental manipulations and looking beyond HF frequency to explore relationships between sleep and HFs: in a controlled model of new-onset HFs, HFs were linked with more PSG awakenings, more WASO, and more light Stage N1 sleep in young premenopausal women treated with a gonadotropinreleasing hormone $(\mathrm{GnRH})$ agonist that simulates menopause; ${ }^{79}$ WASO time increased, on average, by $6.3 \mathrm{~min}$ for each additional HF measured. Furthermore, the majority $(66 \%)$ of HFs occurred during wake or Stage N1 sleep (few HFs occurred during REM sleep), with no difference in the first and second halves of the night. ${ }^{80}$ We recently developed an index to determine how much wake time was associated with HFs rather than only considering HF frequency: in a group of women in the menopausal transition with mild-tomoderate HFs, HF-associated wake time was responsible for, on average, $27 \%$ of objective WASO, although with wide variability in HF impact between women (Figure 3). ${ }^{69}$ This index correlated with self-reported estimates of wakefulness, showing that women are attuned to awakenings associated with HFs. ${ }^{69}$

In summary, while not all HFs are associated with disturbed sleep and not all wakefulness is associated with HFs, HFs are a strong correlate of poor sleep and many HF events are closely linked with periods of wake measured with PSG. The strong overlap in timing between HF onset 

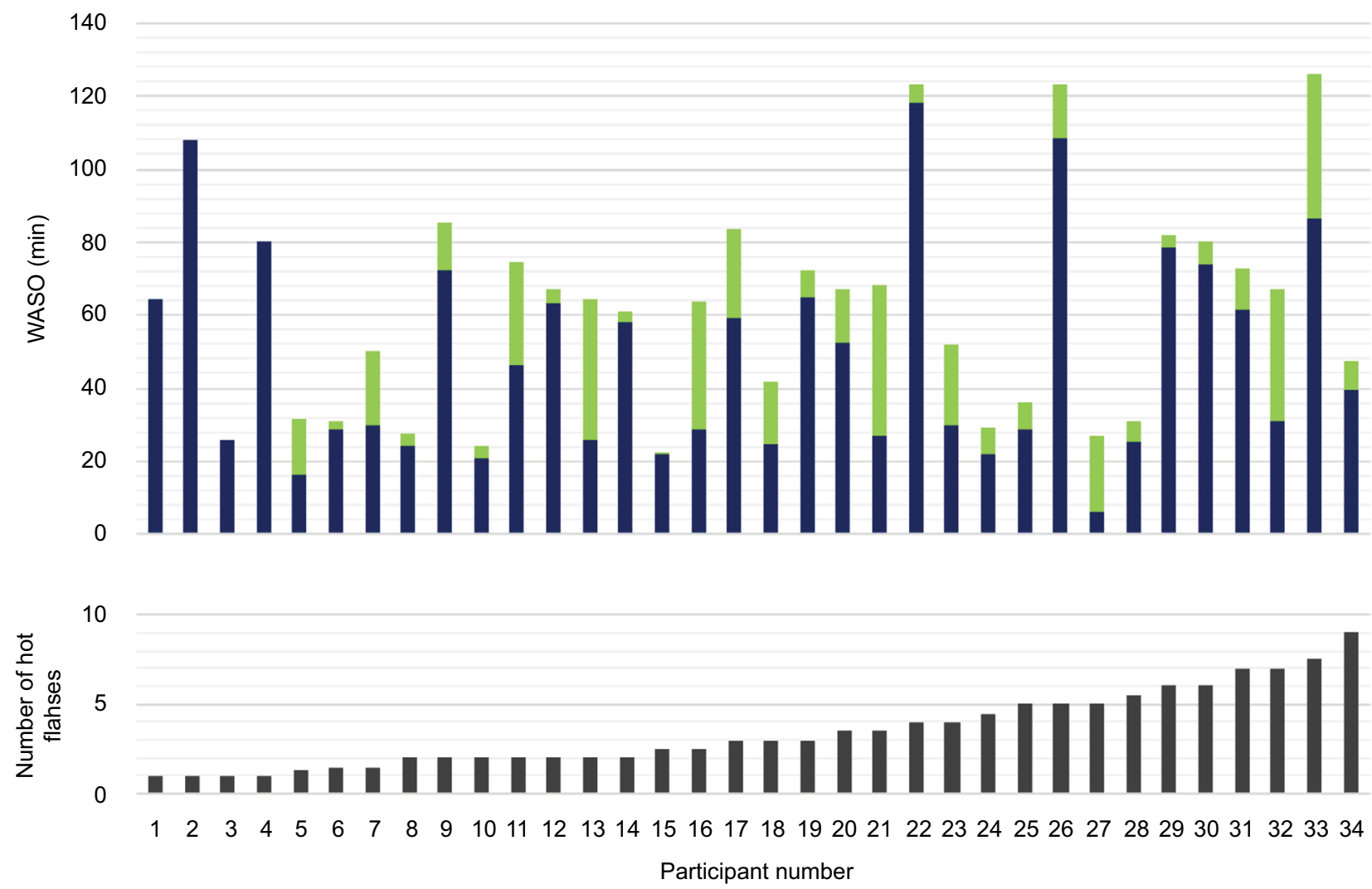

Figure 3 Amount of PSG-defined wakefulness associated with hot flashes relative to total WASO in individual women who presented with varying numbers of hot flashes, as measured from sternal skin conductance, during overnight laboratory stays.

Notes: There was high variability between women in the number of hot flashes measured and in their associated amount of wakefulness. Data from de Zambotti et al. ${ }^{69}$

Abbreviations: PSG, polysomnography; WASO, wakefulness after sleep onset.

and awakenings that we and others have found suggests that these events may be driven by a common mechanism within the CNS in response to fluctuating estrogen levels, although sweating triggered by an HF may still contribute to or extend the interval of waking. ${ }^{69}$ Freedman and colleagues have conducted several studies exploring the mechanism of the HF and hypothesize that declining estradiol levels trigger HFs mediated by central sympathetic nervous system activation. ${ }^{81}$ This group also showed, using fMRI, that there was a greater activation in the anterior insular cortex during the 20 s period immediately before a rise in skin conductance (marking HF onset) compared with the first 20 s of the HF, suggesting that it plays a role in the mechanism of an $\mathrm{HF}^{82}$ How this activation pattern might also relate to an awakening coincident with a HF is not clear but does indicate the relevance of looking at changes in EEG and other physiological measures in advance of the skin conductance rise marking an HF. Nocturnal HFs are an important component of sleep disturbance during midlife, particularly in women with severe sleep difficulties, that qualifies them for insomnia disorder, as discussed later.

\section{Impact of sleep difficulties in the menopausal transition}

Severity and duration of sleep difficulties vary in women transitioning menopause, with some having occasional poor nights of sleep, which are bothersome and transiently impact quality of life. Others have chronic and severe sleep difficulties, associated with greater and more prolonged impact on daytime functioning, quality of life, and health. Insomnia disorder in the general population is linked with adverse mental and physical conditions, ${ }^{83}$ including depression and cardiovascular disease, ${ }^{84}$ and insomnia combined with short sleep duration is proposed as the most biologically severe phenotype of the disorder. ${ }^{85}$ Furthermore, longitudinal data from a population of community-dwelling postmenopausal women specifically linked insomnia with increased vulnerability to physical and mental impairments. ${ }^{86}$ It is unclear if severe sleep difficulties that either develop or are exacerbated, in the context of the menopausal transition, have a similar impact or different impacts on long-term health compared with insomnia developed at other times, and how comorbid 
features such as the presence of severe HFs, depression, and poor physical health might interact with sleep difficulties to contribute to impairment.

Data are emerging from longitudinal studies such as SWAN linking midlife sleep problems with subsequent mental health: women with sleep problems, in late reproductive stage or early in the menopausal transition, are at greater risk for persistent/recurrent depression relative to single episode major depressive disorder (MDD), over and above risk conferred from having a lifetime history of depression. ${ }^{87}$ Recent cross-sectional data also link poor sleep with subclinical cardiovascular risk markers. ${ }^{88,89}$ Poor subjective sleep quality and short sleep duration (from actigraphy) were associated with increased carotid atherosclerosis in peri/postmenopausal women, independent of HFs. ${ }^{88}$ Longitudinal data from SWAN found that lower sleep efficiency was related to increases in resting systolic and diastolic blood pressures and increased risk for incident hypertension across follow-up and lower NREM delta power was associated with greater increase in diastolic blood pressure over time, after adjustment for age and baseline blood pressure. ${ }^{90}$ However, after adjustment for additional factors (including race, BMI, and diabetes mellitus), associations were no longer significant, apart from that between delta power and diastolic blood pressure. We have focused our studies on women with clinically significant sleep complaints (insomnia) developed in the context of the menopausal transition and found that these women have altered autonomic and cardiovascular regulation, including a less-sustained dip in blood pressure across the night relative to women without insomnia. ${ }^{91}$ A less effective "cardiovascular holiday" during the night may have consequences for longterm cardiovascular health, which needs to be investigated in the context of menopausal insomnia in future work.

\section{Sleep and mood disturbance in the menopausal transition}

The bidirectional relationship between sleep and mood disturbances is well established across genders and ages. Up to $90 \%$ individuals with MDD have sleep complaints, ${ }^{92}$ and MDD has also been associated with alterations in objectively measured sleep. ${ }^{93}$ In nonpsychiatric populations, self-reported poor sleep has been consistently associated with symptoms of depression and anxiety, ${ }^{94,95}$ while the association between objectively measured sleep duration/quality and mood symptoms is comparably weaker. ${ }^{96}$

Several longitudinal studies have found that depressive symptoms increase during the menopausal transition. ${ }^{97}$ The associations between sleep and mood disturbances in women going through the menopausal transition are largely consistent with those reported in the general population. Cross-sectional studies with self-report sleep measures have found consistent and strong associations between poor self-report sleep or greater symptoms of insomnia, with higher symptoms of depression $^{33,61,98-105}$ and anxiety. ${ }^{61,99,101,103,106}$ These include large-scale epidemiological studies, those that included women of both Caucasian and non-Caucasian background, and those in premenopausal, menopausal transition, and postmenopausal stages. One study further suggested that sleep initiation problems may be associated with anxiety, while nonrestorative sleep may be associated with depression. ${ }^{103}$

Findings are somewhat less consistent when sleep is measured via objective methods but point to an association between objective sleep disturbance and worse mood. In women with HFs aged $>40$ years, those with depression had shorter sleep duration, longer sleep onset latency, and lower SE (but not number of awakenings or WASO) based on actigraphy compared to those without depression. ${ }^{107}$ In healthy samples, poorer actigraphy-measured sleep was associated with worse mood in menopausal but not in premenopausal women. ${ }^{108}$ In a study of pre-, peri-, and postmenopausal women with subclinical depressive symptoms, Toffol et al found that higher depression scores on the Beck Depression Inventory were associated with lower sleep efficiency and shorter total sleep time in perimenopausal women and with more REM sleep in postmenopausal women, after controlling for age, although effects were lost when sleep-related items of the questionnaire were removed. ${ }^{109}$

Longitudinal studies are rare. One study followed 309 women aged 43-53 years for 5 years and found that depressive symptoms were not related to menopausal status or annual change in estradiol but were associated with HFs and sleep disturbance. ${ }^{110}$ A 13-year longitudinal study on 297 premenopausal women aged 42-52 years from SWAN found that those who experienced persistent/recurrent MDD during annual follow-ups were more likely to have reported sleep problems at baseline. ${ }^{87}$ Therefore, sleep problems during the menopausal transition are not only associated with concurrent symptoms of depression and anxiety but may also be a risk factor for depression in the future.

Mechanisms for the sleep-mood association are not well understood. It is possible that mood disturbances may result from poor sleep via neurobiological ${ }^{111}$ as well as psychological $^{112}$ mechanisms. For example, in perimenopausal women, unhelpful thoughts and beliefs about sleep were found to mediate the relationship between poor sleep and mood disturbances. ${ }^{101}$ It is also possible that sleep and 
mood disturbances are linked via common causes that exert simultaneous influence on both processes. Another potential common cause is age-related changes in circadian regulation. Circadian processes are critical to sleep/wake regulation and play a role in mood and affect. ${ }^{113}$ Consistent with findings in general populations, later circadian phase markers were linked to both higher anxiety ${ }^{114}$ and the presence of depression ${ }^{115}$ in menopausal women.

HFs have also been examined as a potential link with mood disturbances, with some theorizing that HFs disrupt sleep, which in turn leads to daytime mood symptoms (domino effect $)^{116}$ or via bothersomeness of HFs. ${ }^{117}$ However, not all women with HFs develop depression and many midlife women experience depression in the absence of HFs. ${ }^{118}$ More recently, investigators have shown some differentiation between HF-sleep and depression-sleep relationships, at least in postmenopausal women. HFs and depression had an additive effect on self-report sleep disturbance, ${ }^{105}$ and self-report HFs and depression symptoms were associated with different sleep disturbance patterns, with HFs being uniquely associated with frequent awakenings, whereas depression was uniquely associated with difficulty falling asleep and waking up earlier than desired ${ }^{119}$ in a clinical sample of postmenopausal women. Results from a clinical trial further show independence of HFs, mood, and sleep: Joffe et al ${ }^{120}$ found that increasing estradiol (in perimenopausal women) and improved sleep quality (in peri/postmenopausal women) predicted improved mood, but reduced HFs did not. To further investigate the interdependency between sleep, HFs, and mood, Joffe et al ${ }^{121}$ conducted an experimental study that induced HFs in healthy nonmenopausal women. They found that PSG-measured sleep and nighttime HFs independently predicted mood deterioration.

It is worth noting that although sleep disturbance is a significant concern during the menopausal transition and longitudinal changes in menopausal status over time are associated with increased risk of depressive symptoms, the rate of MDD as a psychiatric disorder is similar to that at other times in a woman's reproductive life. ${ }^{122}$ Therefore, proactively treating clinically significant mood disorders, normalizing some degree of mood disturbances, and effectively addressing sleep problems may benefit psychological well-being during the menopausal transition.

\section{Sleep disorders in the context of the menopausal transition Sleep-disordered breathing (SDB)}

Women have an increased risk of SDB after menopause, attributed to aging and menopause-related factors, ${ }^{123-125}$ as reviewed in detail elsewhere., ${ }^{2,126,127}$ In fact, Freedman and Roehrs $^{128}$ found, in a sample of midlife women with sleep complaints, that 53\% had SDB, periodic limb movement disorder, or both and that the apnea-hypopnea index (AHI), periodic limb movement index, and arousals, but not HFs, were predictors of PSG-defined SE. The community-based SWAN study found $20 \%$ prevalence of moderate/severe SDB (>15 apnea + hypopnea events/hour) in midlife women, ${ }^{129}$ substantially higher than reports for younger, premenopausal women (4\%). ${ }^{130}$

Progression through menopausal stages is associated with SDB severity, with a 4\% higher AHI for every additional year since entering the menopausal transition, independent of age, BMI, and neck and waist circumferences, although these factors were also associated with a higher AHI. ${ }^{131}$ The decline in estrogen and progesterone after menopause likely plays a role in increased risk for SDB; progesterone increases ventilatory drive and affects upper airway dilatory muscles ${ }^{132}$ and partly mediates its effects through estrogen-dependent receptors; ${ }^{133}$ hence, the menopausal decline in both these hormones could impact respiration. Also, HT is associated with a lower prevalence of SDB, ${ }^{123,125}$ although clinical trials of HT have produced conflicting results. ${ }^{134} \mathrm{~A}$ change in body habitus, with increased weight gain and an accumulation of adipose tissue in the upper part of the body as well as a shift toward visceral adipose deposition as women progress through the menopausal transition, is also a critical risk factor for SDB after menopause. ${ }^{125,131}$

Given the health risks associated with untreated SDB, women suspected of having the disorder should be formally evaluated and treated; continuous positive airway pressure (CPAP) remains the treatment of choice for SDB in peri- and postmenopausal women.

\section{Insomnia disorder}

Epidemiological studies have varied in their assessment of the presence and severity of sleep difficulties, and while there is a clear increase in poor sleep quality as women transition menopause, it is important to distinguish who experiences severe and persistent sleep disturbance associated with daytime impairment, qualifying them for insomnia disorder (Table 2). Based on a phone interview of 982 women, Ohayon ${ }^{37}$ reported that $26 \%$ of perimenopausal women qualified for a DSM-IV diagnosis of insomnia (with insomnia duration set at 6 months rather than 1 month), with difficulty maintaining sleep being the most common symptom. Some women had preexisting insomnia symptoms before entering the menopausal transition, but one-third $(31.8 \%)$ reported that their insomnia symptoms were related to the development of menopause. ${ }^{37}$ 
Table 2 DSM 5 criteria for insomnia disorder

- Dissatisfaction with sleep quantity or quality, with difficulty initiating or maintaining sleep, and/or early-morning awakening

- Sleep disturbance causes significant distress or impairment in social, occupational, educational, academic, or behavioral functioning

- Sleep difficulty occurs $\geq 3$ nights per week, for $\geq 3$ months, despite adequate opportunity for sleep

- Insomnia does not co-occur with another sleep disorder

Notes: DSM-IV criteria (used in studies until recently) specified a duration of at least I month and distinguished between primary and secondary insomnia. Data from DSM 5. ${ }^{35}$ Abbreviations: DSM 5, Diagnostic and Statistical Manual of Mental Disorders. 5th edition.

A major factor in the increased prevalence of insomnia disorder in midlife women was HFs; experiencing HFs (mild, moderate, or severe) was strongly associated with a diagnosis of insomnia, with the likelihood of having chronic insomnia symptoms increasing with the severity of HFs. ${ }^{37}$ Similarly, HF severity was a significant factor associated with high insomnia severity index scores in midlife women. ${ }^{136}$ Some women may be more vulnerable to the development of insomnia disorder in the context of the menopausal transition; we found that women with first-onset insomnia disorder relative to controls, all in the menopausal transition, were more likely to have obsessive-compulsive personality disorder, a past depressive episode, and a history of severe premenstrual symptoms. ${ }^{137}$ Also, higher neuroticism (the general tendency to experience negative affect such as fear, sadness, anxiety, embarrassment, anger, and guilt) along with greater interference from HFs (eg, with work and social activities), was associated with insomnia classification. ${ }^{137}$ As shown with prospective SWAN data, chronic stress exposure also predisposes midlife women to insomnia. ${ }^{138}$ In the context of the menopausal transition, therefore, classical clinical features of insomnia such as elevated rumination, anxiety, generalized hyperarousal, stress reactivity, and neuroticism ${ }^{139-141}$ seem to overlap with aspects of insomnia specific to the menopausal transition, such as HFs.

While studies have not linked gross disturbances in PSG-derived sleep measures with menopausal stages in population studies of midlife women (despite subjective sleep difficulties), we found stark differences in PSG measures in women with, relative to women without, insomnia disorder developed in the menopausal transition. ${ }^{142}$ Women meeting DSM-IV criteria for insomnia had a poorer SE, more WASO, and shorter TST, with almost $50 \%$ of them having $<6$ hours of TST during a laboratory PSG study. Importantly, women with insomnia were more likely to have objective HFs and the presence of HFs predicted the number of PSG-awakenings per hour of sleep, ${ }^{142}$ supporting the critical role of HFs in insomnia in the menopausal transition context. ${ }^{37}$ Based on sleep diaries, women with insomnia also reported a shorter TST, with more awakenings and more HFs compared to women without insomnia. ${ }^{142}$ In an interesting comparison of peri/postmenopausal and premenopausal women with insomnia (all aged between 40 and 59 years), $\mathrm{Xu}$ et al ${ }^{143}$ found that subjective measures, including scores on the insomnia severity index, Beck depression inventory, and sleep diary measures, were similar between the two groups but that peri/postmenopausal women with insomnia had a longer PSG-defined total wake time and lower SE (81.8\% vs $86.0 \%)$. While this study has some limitations since the presence of HFs was not considered and insomnia did not necessarily develop in the menopausal transition, results suggest that PSG measures of sleep quality are impacted to a greater extent in peri/postmenopausal than in premenopausal women with insomnia.

Chronic stress could add a layer of complexity to PSG sleep disturbance in insomnia disorder in midlife women - Hall et al ${ }^{138}$ found that women with more chronic stress exposure over a 9-year period had greater PSG-assessed WASO (as well as being more likely to have insomnia) than participants with moderate stress exposure. We also found that women with insomnia showed less within-night recovery in autonomic vagal functioning following pre-sleep acute stress exposure ${ }^{144}$ as well as raised $\mathrm{HR}^{145}$ and raised blood pressure ${ }^{91}$ during sleep relative to women without insomnia, also in the menopausal transition.

Taken together, PSG studies focused on women with insomnia disorder in the menopausal transition show evidence of disrupted sleep, partly in association with HFs, as well as less efficient autonomic and cardiovascular recovery during sleep compared to women without insomnia disorder. No matter what the precipitating factors for insomnia are, behavioral conditioning and sleep-associated behaviors may prolong insomnia; ${ }^{127}$ these behaviors need to be addressed in order to successfully treat insomnia.

\section{Management of insomnia in the menopausal transition}

Insomnia in the context of the menopausal transition may be attributed to multiple overlapping factors, making treatment challenging and requiring a flexible and individualized response. Assessment procedures and treatment options for insomnia in the context of the menopausal transition are pro- 

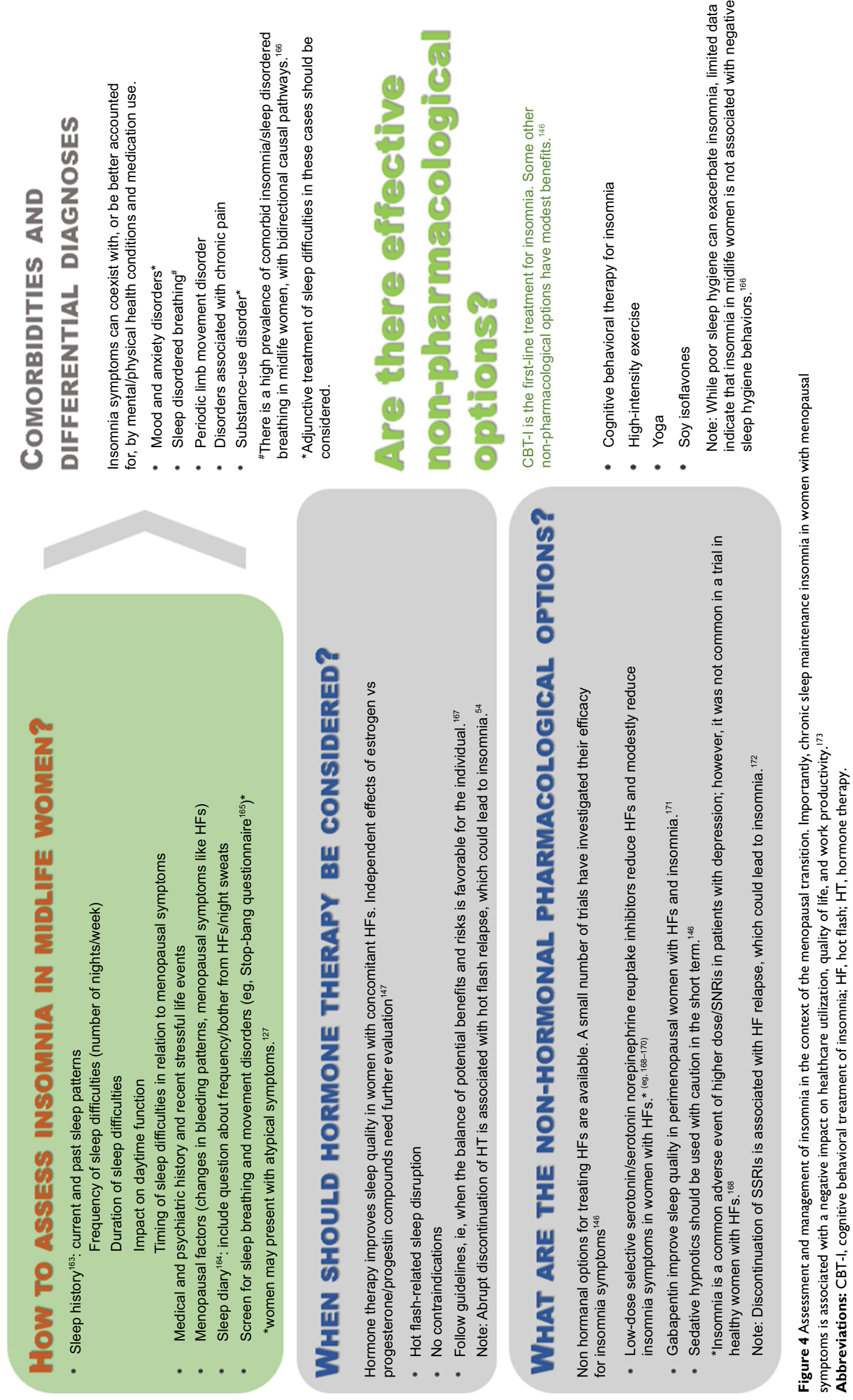
vided in Figure 4. In some cases, combined treatments may be required, such as for women who have severe HFs, sleep problems, and depression. Options include HT, nonhormonal pharmacological medications, and behavioral interventions. Randomized clinical trials have shown that successful treatment of severe HFs with HT, and more recently, with selective serotonin/serotonin norepinephrine reuptake inhibitors, also leads to improvements in sleep quality, although the side effect profiles of these medications need to be carefully considered before use. ${ }^{2,146,147}$ Dietary supplements for menopausal symptoms have also been investigated. Soy isoflavones, for example, have been shown in randomized controlled trials to reduce menopausal symptoms ${ }^{148}$, including self-reported sleep disturbance. ${ }^{149,150}$

Cognitive behavioral treatment of insomnia (CBT-I), considered the primary intervention for all patients with chronic insomnia, ${ }^{151}$ should be similarly considered for women with insomnia in the menopausal transition. CBT-I typically includes sleep hygiene to improve sleep conditions, sleep restriction to increase sleep efficiency, stimulus control focused to enhance the association between bed and sleep, and cognitive restructuring to reduce dysfunctional beliefs and attitudes about sleep. In nonmenopausal populations, CBT-I approaches consistently improve sleep, ${ }^{152}$ including when sleep disturbance and insomnia are comorbid with other mental and physical health conditions. ${ }^{153}$ Compared to sleep medication alone, effects of CBT-I are comparable in the short term and superior in the long term. ${ }^{154}$ However, CBT-I has only recently been evaluated specifically for insomnia during the menopausal transition. McCurry et a ${ }^{155}$ conducted a randomized clinical trial on 106 peri- and postmenopausal women with insomnia symptoms and $\geq 2$ HFs daily, comparing 8-week telephone-administered CBT-I with menopause education as control. Compared to the control condition, the CBT-I condition had significantly greater reduction in insomnia symptoms and greater improvements in selfreport sleep quality; these improvements were maintained at 6 months posttreatment and were clinically meaningful. ${ }^{155}$ Interestingly, there were no group differences in ratings of HF frequency (day or night), severity, or bother, although HF-related interference was reduced, mostly due to a reduction in sleep-related interference from HFs, ${ }^{155}$ suggesting that women may still have woken up in association with HFs but were able to return to sleep faster in the treatment group. Preliminary data from an open trial of CBT-I in women whose sleep was affected by menopause also indicated a significant reduction in insomnia (and depression) symptoms posttreatment. ${ }^{127}$ These promising findings provide empirical evidence for future implementation and dissemination of CBT-I in menopausal populations.

A factor that has not yet been addressed for insomnia in the context of the menopausal transition is whether interventions may be applied in advance of menopausal symptoms to protect against insomnia. In the context of insomnia in general, it has been suggested that it would be beneficial to identify individuals vulnerable to insomnia and provide primary prevention (eg, education on stress management) and secondary prevention (eg, modify sleep-related behaviors) to prevent relapse or maintenance of insomnia. ${ }^{156}$ Similarly, women vulnerable to insomnia in the context of the menopausal transition (eg, history of severe premenstrual syndrome and neurotic personality style ${ }^{137}$ ) may benefit from preventative interventions. Also, certain behaviors may protect against sleep problems; high levels of recreational physical activity are associated with better sleep in midlife women. ${ }^{157}$

\section{Conclusion}

Sleep difficulties increase in prevalence as women transition menopause. For some women, sleep problems are severe and impact daytime functioning and quality of life and may have long-term consequences for mental and physical health. Further research is needed to better understand factors that contribute to the development of sleep disturbance in the context of the menopausal transition, as described in Box 2. HFs are a unique aspect of insomnia in the menopausal transition and are strongly associated with reports of disrupted sleep, and HF-associated wakefulness makes a significant contribution to the amount of PSG-measured wakefulness during the night. In addition, given the co-occurrence of HFs and awakenings, women attribute those awakenings as specifically caused by HFs. Since some women may have sleep problems independent of HFs, other factors directly related to the menopausal transition (eg, instability/changes in the hormone environment with progressive decreases in estradiol and increases in FSH) and/or coincident with the transition (eg, SDB or movement disorders, mood disturbance, presence of a medical condition, and life stressors) also need to be considered. It, therefore, is critical to assess insomnia symptoms in the context of menopause as well as considering physical and mental health and presence of stressful life events. Given the presence of unique sleepdisruptive factors (eg, HFs) and the multifactorial nature of sleep difficulties in women approaching menopause, with multiple factors often interacting, treatment needs to be tailored for women. 


\section{Box 2 Research agenda}

- Future studies about sleep across the menopausal transition should apply STRAW criteria9 to standardize the definitions of menopausal stages.

- More longitudinal studies are needed to track changes in sleep PSG and EEG markers in relation to changes in reproductive hormone levels as well as symptoms such as HFs, over time. Analyses should consider subgroups of women who might be more vulnerable to the hormonal changes of the menopausal transition (eg, past sensitivity to hormone changes such as in premenstrual dysphoric disorder) or who show different trajectories of HFs (eg, those with persistently high and early onset HFs, who have a more adverse health profile). ${ }^{51}$

- Given the often discordant results between subjective and objective assessments of sleep quality and HFs, both types of assessment should be included to better understand what factors influence physiology and/or symptom perception, which can better inform treatment.

- Consider tracking sleep with standard actigraphy in larger groups of women and for longer periods of time than is possible with PSG in relation to symptoms such as HFs (measured with ambulatory devices) and hormone levels sampled frequently using saliva. Also, access to "big data" about behaviors (eg, menstrual cycle patterns, sleep, menopausal symptoms, and stress), which can be obtained via consumer sleep devices (eg, wearables) and mobile technology, may ultimately provide new insight into the hormone-sleep-menopause relationship.

- Longitudinal studies are needed to track trajectories in PSG and self-report sleep measures in women who approach the menopausal transition without insomnia, who then develop insomnia, including evaluation of HFs, and to investigate whether treatment effectively corrects sleep disruption and aberrant physiological features in these women. Also, studies should track women with preexisting insomnia to determine whether the menopausal transition exacerbates their symptoms. Finally, studies are needed to investigate whether CBT-I is effective in women across the range in the severity of HFs and their impact on sleep (Figure 3 ) to determine in what cases CBT-I alone vs combined with other treatments should be considered.

- Clarity is still needed about the relationship between measured HFs and sleep disturbance, whether the relationship differs across individuals, time of night, or in relation to HF severity or bother, and whether there is a common central or autonomic mechanism underlying their association. Interventions that increase/decrease HFs may be best suited to determine underlying mechanisms of the HF-wake relationship. Also, more refined analysis, such as using the changes that occur in autonomic measures just before and coincident with HFs, ${ }^{158}$ may be useful to more precisely align EEG-defined wake and HF events in future work.

Abbreviations: STRAW, Stages of Reproductive Aging Workshop; PSG, polysomnography; HF, hot flash; CBT-I, cognitive behavioral treatment of insomnia; EEG, electroencephalograph.

\section{Acknowledgments}

This study is supported by the National Institutes of Health, Grant HL103688 (FCB). The content is solely the responsibility of the authors and does not necessarily represent the official views of the National Institutes of Health.

\section{Disclosure}

The authors report no conflicts of interest in this work.

\section{References}

1. Ameratunga D, Goldin J, Hickey M. Sleep disturbance in menopause. Intern Med J. 2012;42(7):742-747.

2. Joffe H, Massler A, Sharkey KM. Evaluation and management of sleep disturbance during the menopause transition. Semin Reprod Med. 2010;28(5):404-421.

3. Nowakowski S, Meliska CJ, Martinez LF, Parry BL. Sleep and menopause. Curr Neurol Neurosci Rep. 2009;9(2):165-172.

4. Polo-Kantola P. Sleep problems in midlife and beyond. Maturitas. 2011;68(3):224-232.

5. Shaver JL, Woods NF. Sleep and menopause: a narrative review. Menopause. 2015;22(8):899-915.

6. Burger HG, Hale GE, Dennerstein L, Robertson DM. Cycle and hormone changes during perimenopause: the key role of ovarian function. Menopause. 2008;15(4 Pt 1):603-612.

7. Santoro N. The menopausal transition. Am J Med. 2005;118(Suppl 12B):8-13.

8. Soules MR, Sherman S, Parrott E, et al. Executive summary: stages of Reproductive Aging Workshop (STRAW). Fertil Steril. 2001;76(5):874-878.

9. Harlow SD, Gass M, Hall JE, et al. Executive summary of the Stages of Reproductive Aging Workshop + 10: addressing the unfinished agenda of staging reproductive aging. J Clin Endocrinol Metab. 2012;97(4):1159-1168
10. Dennerstein L, Lehert P, Burger HG, Guthrie JR. New findings from non-linear longitudinal modelling of menopausal hormone changes. Hum Reprod Update. 2007;13(6):551-557.

11. Taffe JR, Dennerstein L. Menstrual patterns leading to the final menstrual period. Menopause. 2002;9(1):32-40.

12. Van Voorhis BJ, Santoro N, Harlow S, Crawford SL, Randolph $\mathrm{J}$. The relationship of bleeding patterns to daily reproductive hormones in women approaching menopause. Obstet Gynecol. 2008;112(1):101-108.

13. Hale GE, Zhao X, Hughes CL, Burger HG, Robertson DM, Fraser IS. Endocrine features of menstrual cycles in middle and late reproductive age and the menopausal transition classified according to the Staging of Reproductive Aging Workshop (STRAW) staging system. J Clin Endocrinol Metab. 2007;92(8):3060-3067.

14. McKinlay SM, Brambilla DJ, Posner JG. The normal menopause transition. Maturitas. 1992;14(2):103-115.

15. Bacon JL. The menopausal transition. Obstet Gynecol Clin North Am. 2017;44(2):285-296.

16. Gallicchio L, Whiteman MK, Tomic D, Miller KP, Langenberg P, Flaws JA. Type of menopause, patterns of hormone therapy use, and hot flashes. Fertil Steril. 2006;85(5):1432-1440.

17. Kravitz HM, Ganz PA, Bromberger J, Powell LH, Sutton-Tyrrell K Meyer PM. Sleep difficulty in women at midlife: a community survey of sleep and the menopausal transition. Menopause. 2003;10(1): $19-28$.

18. Xu Q, Lang CP. Examining the relationship between subjective sleep disturbance and menopause: a systematic review and meta-analysis. Menopause. 2014;21(12):1301-1318.

19. Berecki-Gisolf J, Begum N, Dobson AJ. Symptoms reported by women in midlife: menopausal transition or aging? Menopause. 2009;16(5):1021-1029.

20. Dennerstein L, Dudley EC, Hopper JL, Guthrie JR, Burger HG. A prospective population-based study of menopausal symptoms. Obstet Gynecol. 2000;96(3):351-358.

21. Freeman EW, Sammel MD, Gross SA, Pien GW. Poor sleep in relation to natural menopause: a population-based 14-year follow-up of midlife women. Menopause. 2015;22(7):719-726. 
22. Kravitz HM, Zhao X, Bromberger JT, et al. Sleep disturbance during the menopausal transition in a multi-ethnic community sample of women. Sleep. 2008;31(7):979-990.

23. Pien GW, Sammel MD, Freeman EW, Lin H, DeBlasis TL. Predictors of sleep quality in women in the menopausal transition. Sleep. 2008;31(7):991-999.

24. Tom SE, Kuh D, Guralnik JM, Mishra GD. Self-reported sleep difficulty during the menopausal transition: results from a prospective cohort study. Menopause. 2010;17(6):1128-1135.

25. Woods NF, Mitchell ES. Sleep symptoms during the menopausal transition and early postmenopause: observations from the Seattle Midlife Women's Health Study. Sleep. 2010;33(4):539-549.

26. Kuh DL, Hardy R, Wadsworth M. Women's health in midlife: the influence of the menopause, social factors and health in earlier life. Br J Obstet Gynaecol. 1997;104(12):1419.

27. Leon-Ferre RA, Majithia N, Loprinzi CL. Management of hot flashes in women with breast cancer receiving ovarian function suppression. Cancer Treat Rev. 2017;52:82-90.

28. Moon Z, Hunter MS, Moss-Morris R, Hughes LD. Factors related to the experience of menopausal symptoms in women prescribed tamoxifen. J Psychosom Obstet Gynaecol. 2017;38(3):226-235.

29. Chang HY, Jotwani AC, Lai YH, et al. Hot flashes in breast cancer survivors: frequency, severity and impact. Breast. 2016;27:116-121.

30. Otte JL, Carpenter JS, Russell KM, Bigatti S, Champion VL. Prevalence, severity, and correlates of sleep-wake disturbances in longterm breast cancer survivors. J Pain Symptom Manage. 2010;39(3): 535-547.

31. Savard MH, Savard J, Caplette-Gingras A, Ivers H, Bastien C. Relationship between objectively recorded hot flashes and sleep disturbances among breast cancer patients: investigating hot flash characteristics other than frequency. Menopause. 2013;20(10):997-1005.

32. LeBlanc M, Merette C, Savard J, Ivers H, Baillargeon L, Morin CM. Incidence and risk factors of insomnia in a population-based sample. Sleep. 2009;32(8):1027-1037.

33. Moreno-Frias C, Figueroa-Vega N, Malacara JM. Relationship of sleep alterations with perimenopausal and postmenopausal symptoms. Menopause. 2014;21(9):1017-1022.

34. Sowers MF, Zheng H, Kravitz HM, et al. Sex steroid hormone profiles are related to sleep measures from polysomnography and the Pittsburgh Sleep Quality Index. Sleep. 2008;31(10):1339-1349.

35. Hollander LE, Freeman EW, Sammel MD, Berlin JA, Grisso JA, Battistini M. Sleep quality, estradiol levels, and behavioral factors in late reproductive age women. Obstet Gynecol. 2001;98(3):391-397.

36. Lampio L, Saaresranta T, Engblom J, Polo O, Polo-Kantola P. Predictors of sleep disturbance in menopausal transition. Maturitas. 2016;94:137-142.

37. Ohayon MM. Severe hot flashes are associated with chronic insomnia. Arch Intern Med. 2006;166(12):1262-1268.

38. Shaver JL, Giblin E, Paulsen V. Sleep quality subtypes in midlife women. Sleep. 1991;14(1):18-23.

39. Walters JF, Hampton SM, Ferns GA, Skene DJ. Effect of menopause on melatonin and alertness rhythms investigated in constant routine conditions. Chronobiol Int. 2005;22(5):859-872.

40. de Zambotti M, Willoughby AR, Sassoon SA, Colrain IM, Baker FC. Menstrual cycle-related variation in physiological sleep in women in the early menopausal transition. J Clin Endocrinol Metab. 2015;100(8):2918-2926.

41. Kravitz HM, Janssen I, Santoro N, et al. Relationship of day-to-day reproductive hormone levels to sleep in midlife women. Arch Intern Med. 2005;165(20):2370-2376.

42. Kaplan KA, Hardas PP, Redline S, Zeitzer JM, Sleep Heart Health Study Research Group. Correlates of sleep quality in midlife and beyond: a machine learning analysis. Sleep Med. 2017;34:162-167.

43. Young T, Rabago D, Zgierska A, Austin D, Laurel F. Objective and subjective sleep quality in premenopausal, perimenopausal, and postmenopausal women in the Wisconsin Sleep Cohort Study. Sleep. 2003;26(6):667-672.
44. Campbell IG, Bromberger JT, Buysse DJ, et al. Evaluation of the association of menopausal status with delta and beta EEG activity during sleep. Sleep. 2011;34(11):1561-1568.

45. Lampio L, Polo-Kantola P, Himanen SL, et al. Sleep during menopausal transition: a 6-year follow-up. Sleep. 2017;40(7). doi: 10.1093/sleep/ zsx090.

46. de Zambotti M, Colrain IM, Baker FC. Interaction between reproductive hormones and physiological sleep in women. J Clin Endocrinol Metab. 2015;100(4):1426-1433.

47. Mong JA, Cusmano DM. Sex differences in sleep: impact of biological sex and sex steroids. Philos Trans $R$ Soc Lond B Biol Sci. 2016;371(1688):20150110.

48. Avis NE, Crawford SL, Greendale G, et al. Duration of menopausal vasomotor symptoms over the menopause transition. JAMA Intern Med. 2015;175(4):531-539.

49. Gold EB, Colvin A, Avis N, et al. Longitudinal analysis of the association between vasomotor symptoms and race/ethnicity across the menopausal transition: study of Women's Health across the Nation. Am J Public Health. 2006;96(7):1226-1235.

50. Woods NF, Mitchell ES, Landis C. Anxiety, hormonal changes, and vasomotor symptoms during the menopause transition. Menopause. 2005;12(3):242-245.

51. Tepper PG, Brooks MM, Randolph JF Jr, et al. Characterizing the trajectories of vasomotor symptoms across the menopausal transition. Menopause. 2016;23(10):1067-1074.

52. Kronenberg F. Menopausal hot flashes: a review of physiology and biosociocultural perspective on methods of assessment. J Nutr. 2010;140(7):1380S-1385S.

53. Freedman RR. Hot flashes: behavioral treatments, mechanisms, and relation to sleep. Am J Med. 2005;118(Suppl 12B):124-130.

54. Mishra GD, Dobson AJ. Using longitudinal profiles to characterize women's symptoms through midlife: results from a large prospective study. Menopause. 2012;19(5):549-555.

55. Mishra GD, Kuh D. Health symptoms during midlife in relation to menopausal transition: British prospective cohort study. BMJ. 2012;344:e402.

56. Thurston RC, El Khoudary SR, Tepper PG, et al. Trajectories of vasomotor symptoms and carotid intima media thickness in the study of Women's Health across the Nation. Stroke. 2016;47(1):12-17.

57. Archer DF, Sturdee DW, Baber R, et al. Menopausal hot flushes and night sweats: where are we now? Climacteric. 2011;14(5):515-528.

58. Freedman RR. Pathophysiology and treatment of menopausal hot flashes. Semin Reprod Med. 2005;23(2):117-125.

59. Freedman RR. Menopausal hot flashes: mechanisms, endocrinology, treatment. J Steroid Biochem Mol Biol. 2014;142:115-120.

60. Rance NE, Dacks PA, Mittelman-Smith MA, Romanovsky AA, Krajewski-Hall SJ. Modulation of body temperature and LH secretion by hypothalamic KNDy (kisspeptin, neurokinin B and dynorphin) neurons: a novel hypothesis on the mechanism of hot flushes. Front Neuroendocrinol. 2013;34(3):211-227.

61. Blumel JE, Cano A, Mezones-Holguin E, et al. A multinational study of sleep disorders during female mid-life. Maturitas. 2012;72(4):359-366.

62. Kravitz HM, Joffe H. Sleep during the perimenopause: a SWAN story. Obstet Gynecol Clin North Am. 2011;38(3):567-586.

63. Xu H, Thurston RC, Matthews KA, et al. Are hot flashes associated with sleep disturbance during midlife? Results from the STRIDE cohort study. Maturitas. 2012;71(1):34-38.

64. Moe KE. Hot flashes and sleep in women. Sleep Med Rev. 2004; $8(6): 487-497$.

65. Pinkerton JV, Abraham L, Bushmakin AG, Cappelleri JC, Komm BS. Relationship between changes in vasomotor symptoms and changes in menopause-specific quality of life and sleep parameters. Menopause. 2016;23(10):1060-1066.

66. Freeman EW, Sammel MD, Sanders RJ. Risk of long-term hot flashes after natural menopause: evidence from the Penn Ovarian Aging Study cohort. Menopause. 2014;21(9):924-932. 
67. Mann E, Hunter MS. Concordance between self-reported and sternal skin conductance measures of hot flushes in symptomatic perimenopausal and postmenopausal women: a systematic review. Menopause. 2011;18(6):709-722.

68. Thurston RC, Blumenthal JA, Babyak MA, Sherwood A. Association between hot flashes, sleep complaints, and psychological functioning among healthy menopausal women. Int J Behav Med. 2006;13(2):163-172.

69. de Zambotti M, Colrain IM, Javitz HS, Baker FC. Magnitude of the impact of hot flashes on sleep in perimenopausal women. Fertil Steril. 2014;102(6):1708.e-1715.e.

70. Polo-Kantola P, Erkkola R, Irjala K, Pullinen S, Virtanen I, Polo O. Effect of short-term transdermal estrogen replacement therapy on sleep: a randomized, double-blind crossover trial in postmenopausa women. Fertil Steril. 1999;71(5):873-880.

71. Sharkey KM, Bearpark HM, Acebo C, Millman RP, Cavallo A, Carskadon MA. Effects of menopausal status on sleep in midlife women. Behav Sleep Med. 2003;1(2):69-80.

72. Shaver J, Giblin E, Lentz M, Lee K. Sleep patterns and stability in perimenopausal women. Sleep. 1988;11(6):556-561.

73. Kravitz HM, Avery E, Sowers M, et al. Relationships between menopausal and mood symptoms and EEG sleep measures in a multiethnic sample of middle-aged women: the SWAN sleep study. Sleep. 2011;34(9):1221-1232.

74. Woodward S, Freedman RR. The thermoregulatory effects of menopausal hot flashes on sleep. Sleep. 1994;17(6):497-501.

75. Erlik Y, Tataryn IV, Meldrum DR, Lomax P, Bajorek JG, Judd HL. Association of waking episodes with menopausal hot flushes. JAMA. 1981;245(17):1741-1744.

76. Freedman RR, Roehrs TA. Lack of sleep disturbance from menopausal hot flashes. Fertil Steril. 2004;82(1):138-144.

77. Freedman RR, Roehrs TA. Effects of REM sleep and ambient temperature on hot flash-induced sleep disturbance. Menopause. 2006;13(4):576-583.

78. Gonen R, Sharf M, Laive P. The association between mid-sleep waking episodes and hot flushes in post-menopausal women. JPsychosomatic Obstet Gynecol. 1986;5:113-117.

79. Joffe H, Crawford S, Economou N, et al. A gonadotropin-releasing hormone agonist model demonstrates that nocturnal hot flashes interrupt objective sleep. Sleep. 2013;36(12):1977-1985.

80. Bianchi MT, Kim S, Galvan T, White DP, Joffe H. Nocturnal hot flashes: relationship to objective awakenings and sleep stage transitions. J Clin Sleep Med. 2016;12(7):1003-1009.

81. Freedman RR. Physiology of hot flashes. Am J Hum Biol. 2001;13(4): 453-464.

82. Freedman RR, Benton MD, Genik RJ 2nd, Graydon FX. Cortical activation during menopausal hot flashes. Fertil Steril. 2006;85(3): 674-678.

83. Buysse DJ. Insomnia. JAMA. 2013;309(7):706-716.

84. Sofi F, Cesari F, Casini A, Macchi C, Abbate R, Gensini GF. Insomnia and risk of cardiovascular disease: a meta-analysis. Eur J Prev Cardiol. 2014;21(1):57-64.

85. Vgontzas AN, Fernandez-Mendoza J, Liao D, Bixler EO. Insomnia with objective short sleep duration: the most biologically severe phenotype of the disorder. Sleep Med Rev. 2013;17(4):241-254.

86. Zaslavsky O, LaCroix AZ, Hale L, Tindle H, Shochat T. Longitudinal changes in insomnia status and incidence of physical, emotional, or mixed impairment in postmenopausal women participating in the Women's Health Initiative (WHI) study. Sleep Med 2015;16(3):364-371.

87. Bromberger JT, Kravitz HM, Youk A, Schott LL, Joffe H. Patterns of depressive disorders across 13 years and their determinants among midlife women: SWAN mental health study. J Affect Disord. 2016;206:31-40.

88. Thurston RC, Chang Y, von Kanel R, et al. Sleep characteristics and carotid atherosclerosis among midlife women. Sleep. 2017;40(2). doi: 10.1093/sleep/zsw052.
89. Matthews KA, Everson-Rose SA, Kravitz HM, Lee L, Janssen I, Sutton-Tyrrell K. Do reports of sleep disturbance relate to coronary and aortic calcification in healthy middle-aged women? Study of Women's Health across the Nation. Sleep Med. 2013;14(3):282-287.

90. Matthews KA, Chang Y, Kravitz HM, et al. Sleep and risk for high blood pressure and hypertension in midlife women: the SWAN (study of Women's Health across the Nation) sleep study. Sleep Med. 2014;15(2):203-208.

91. de Zambotti M, Trinder J, Javitz H, Colrain IM, Baker FC. Altered nocturnal blood pressure profiles in women with insomnia disorder in the menopausal transition. Menopause. 2017;24(3):278-287.

92. Tsuno N, Besset A, Ritchie K. Sleep and depression. J Clin Psychiatry. 2005;66(10):1254-1269.

93. Benca RM, Obermeyer WH, Thisted RA, Gillin JC. Sleep and psychiatric disorders. A meta-analysis. Arch Gen Psychiatry. 1992;49(8):651668. Discussion 669-670.

94. Coo Calcagni S, Bei B, Milgrom J, Trinder J. The relationship between sleep and mood in first-time and experienced mothers. Behav Sleep Med. 2012;10(3):167-179.

95. Rosen IM, Gimotty PA, Shea JA, Bellini LM. Evolution of sleep quantity, sleep deprivation, mood disturbances, empathy, and burnout among interns. Acad Med. 2006;81(1):82-85.

96. Bei B, Milgrom J, Ericksen J, Trinder J. Subjective perception of sleep, but not its objective quality, is associated with immediate postpartum mood disturbances in healthy women. Sleep. 2010;33(4):531-538.

97. Bromberger JT, Kravitz HM. Mood and menopause: findings from the Study of Women's Health across the Nation (SWAN) over 10 years. Obstet Gynecol Clin North Am. 2011;38(3):609-625.

98. Brown JP, Gallicchio L, Flaws JA, Tracy JK. Relations among menopausal symptoms, sleep disturbance and depressive symptoms in midlife. Maturitas. 2009;62(2):184-189.

99. Hartz A, Ross JJ, Noyes R, Williams P. Somatic symptoms and psychological characteristics associated with insomnia in postmenopausal women. Sleep Med. 2013;14(1):71-78.

100. Hsu HC, Lin MH. Exploring quality of sleep and its related factors among menopausal women. J Nurs Res. 2005;13(2):153-164.

101. Kloss JD, Twedy K, Gilrain K. Psychological factors associated with sleep disturbance in menopausal women. Behav Sleep Med. 2004;2(4):177-190.

102. Shin C, Lee S, Lee T, et al. Prevalence of insomnia and its relationship to menopausal status in middle-aged Korean women. Psychiatry Clin Neurosci. 2005;59(4):395-402.

103. Terauchi M, Hiramitsu S, Akiyoshi M, et al. Associations between anxiety, depression and insomnia in peri- and post-menopausal women. Maturitas. 2012;72(1):61-65.

104. Timur S, Sahin NH. Effects of sleep disturbance on the quality of life of Turkish menopausal women: a population-based study. Maturitas. 2009;64(3):177-181.

105. Zervas IM, Lambrinoudaki I, Spyropoulou AC, et al. Additive effect of depressed mood and vasomotor symptoms on postmenopausal insomnia. Menopause. 2009;16(4):837-842.

106. Cheng MH, Hsu CY, Wang SJ, Lee SJ, Wang PH, Fuh JL. The relationship of self-reported sleep disturbance, mood, and menopause in a community study. Menopause. 2008;15(5):958-962.

107. Joffe H, Soares CN, Thurston RC, White DP, Cohen LS, Hall JE. Depression is associated with worse objectively and subjectively measured sleep, but not more frequent awakenings, in women with vasomotor symptoms. Menopause. 2009;16(4):671-679.

108. Baker A, Simpson S, Dawson D. Sleep disruption and mood changes associated with menopause. J Psychosom Res. 1997;43(4): 359-369.

109. Toffol E, Kalleinen N, Urrila AS, et al. The relationship between mood and sleep in different female reproductive states. BMC Psychiatry. 2014;14:177.

110. Avis NE, Crawford S, Stellato R, Longcope C. Longitudinal study of hormone levels and depression among women transitioning through menopause. Climacteric. 2001;4(3):243-249. 
111. Baum KT, Desai A, Field J, Miller LE, Rausch J, Beebe DW. Sleep restriction worsens mood and emotion regulation in adolescents. J Child Psychol Psychiatry. 2014;55(2):180-190.

112. Bei B, Wiley JF, Allen NB, Trinder J. A cognitive vulnerability model on sleep and mood in adolescents under naturalistically restricted and extended sleep opportunities. Sleep. 2015;38(3):453-461.

113. Lewy AJ. Circadian misalignment in mood disturbances. Curr Psychiatry Rep. 2009;11(6):459-465.

114. Toffol E, Kalleinen N, Haukka J, Vakkuri O, Partonen T, Polo-Kantola P. Melatonin in perimenopausal and postmenopausal women: associations with mood, sleep, climacteric symptoms, and quality of life. Menopause. 2014;21(5):493-500.

115. Parry BL, Meliska CJ, Sorenson DL, et al. Increased melatonin and delayed offset in menopausal depression: role of years past menopause, follicle-stimulating hormone, sleep end time, and body mass index. J Clin Endocrinol Metab. 2008;93(1):54-60.

116. Campbell S, Whitehead M. Oestrogen therapy and the menopausal syndrome. Clin Obstet Gynaecol. 1977;4(1):31-47.

117. Thurston RC, Bromberger JT, Joffe H, et al. Beyond frequency: who is most bothered by vasomotor symptoms? Menopause. 2008;15(5):841-847.

118. Thurston RC, Joffe H. Vasomotor symptoms and menopause: findings from the Study of Women's Health across the Nation. Obstet Gynecol Clin North Am. 2011;38(3):489-501.

119. Vousoura E, Spyropoulou AC, Koundi KL, et al. Vasomotor and depression symptoms may be associated with different sleep disturbance patterns in postmenopausal women. Menopause. 2015;22(10):1053-1057.

120. Joffe H, Petrillo LF, Koukopoulos A, et al. Increased estradiol and improved sleep, but not hot flashes, predict enhanced mood during the menopausal transition. J Clin Endocrinol Metab. 2011;96(7):E1044-E1054.

121. Joffe H, Crawford SL, Freeman MP, et al. Independent contributions of nocturnal hot flashes and sleep disturbance to depression in estrogendeprived women. J Clin Endocrinol Metab. 2016;101(10):3847-3855.

122. Judd FK, Hickey M, Bryant C. Depression and midlife: are we overpathologising the menopause? JAffect Disord. 2012;136(3):199-211.

123. Bixler EO, Vgontzas AN, Lin HM, et al. Prevalence of sleep-disordered breathing in women: effects of gender. Am J Respir Crit Care Med. 2001;163(3 Pt 1):608-613.

124. Dancey DR, Hanly PJ, Soong C, Lee B, Hoffstein V. Impact of menopause on the prevalence and severity of sleep apnea. Chest. 2001;120(1):151-155.

125. Young T, Finn L, Austin D, Peterson A. Menopausal status and sleepdisordered breathing in the Wisconsin Sleep Cohort Study. Am J Respir Crit Care Med. 2003;167(9):1181-1185.

126. Collop NA, Adkins D, Phillips BA. Gender differences in sleep and sleep-disordered breathing. Clin Chest Med. 2004;25(2):257-268.

127. Hall MH, Kline CE, Nowakowski S. Insomnia and sleep apnea in midlife women: prevalence and consequences to health and functioning. F1000Prime Rep. 2015;7:63.

128. Freedman RR, Roehrs TA. Sleep disturbance in menopause. Menopause. 2007;14(5):826-829.

129. Hall MH, Matthews KA, Kravitz HM, et al. Race and financial strain are independent correlates of sleep in midlife women: the SWAN sleep study. Sleep. 2009;32(1):73-82.

130. Franklin KA, Sahlin C, Stenlund H, Lindberg E. Sleep apnoea is a common occurrence in females. Eur Respir J. 2013;41(3):610-615.

131. Mirer AG, Young T, Palta M, Benca RM, Rasmuson A, Peppard PE. Sleepdisordered breathing and the menopausal transition among participants in the Sleep in Midlife Women Study. Menopause. 2017;24(2):157-162.

132. Kapsimalis F, Kryger MH. Gender and obstructive sleep apnea syndrome, part 2: mechanisms. Sleep. 2002;25(5):499-506.

133. Bayliss DA, Millhorn DE. Central neural mechanisms of progesterone action: application to the respiratory system. J Appl Physiol (1985). 1992;73(2):393-404

134. Polo-Kantola P. Dealing with menopausal sleep disturbances. Sleep Med Clin. 2008;3:121-131.
135. American Psychiatric Association (APA). Diagnostic and Statistical Manual of Mental Disorders. 5th ed. Washington, DC: APA; 2013.

136. Arakane M, Castillo C, Rosero MF, Penafiel R, Perez-Lopez FR, Chedraui P. Factors relating to insomnia during the menopausal transition as evaluated by the Insomnia Severity Index. Maturitas. 2011;69(2):157-161.

137. Sassoon S, de Zambotti M, Colrain I, Baker F. Association between personality traits and DSM-IV diagnosis of insomnia in peri-and postmenopausal women. Menopause. 2014;21(6):602-611.

138. Hall MH, Casement MD, Troxel WM, et al. Chronic stress is prospectively associated with sleep in midlife women: the SWAN sleep study. Sleep. 2015;38(10):1645-1654.

139. Drake CL, Pillai V, Roth T. Stress and sleep reactivity: a prospective investigation of the stress-diathesis model of insomnia. Sleep. 2014;37(8):1295-1304.

140. Riemann D, Nissen C, Palagini L, Otte A, Perlis ML, Spiegelhalder K. The neurobiology, investigation, and treatment of chronic insomnia. Lancet Neurol. 2015;14(5):547-558.

141. van de Laar M, Verbeek I, Pevernagie D, Aldenkamp A, Overeem $\mathrm{S}$. The role of personality traits in insomnia. Sleep Med Rev. 2010;14(1):61-68.

142. Baker F, Willoughby AR, Sassoon S, Colrain IM, de Zambotti M. Insomnia in women approaching menopause: beyond perception. Psychoneuroendocrinology. 2015;60:96-104.

143. Xu M, Belanger L, Ivers H, Guay B, Zhang J, Morin CM. Comparison of subjective and objective sleep quality in menopausal and nonmenopausal women with insomnia. Sleep Med. 2011;12(1):65-69.

144. de Zambotti M, Sugarbaker D, Trinder J, Colrain IM, Baker FC. Acute stress alters autonomic modulation during sleep in women approaching menopause. Psychoneuroendocrinology. 2016;66:1-10.

145. de Zambotti M, Trinder J, Colrain IM, Baker FC. Menstrual cyclerelated variation in autonomic nervous system functioning in women in the early menopausal transition with and without insomnia disorder. Psychoneuroendocrinology. 2017;75:44-51.

146. Attarian H, Hachul H, Guttuso T, Phillips B. Treatment of chronic insomnia disorder in menopause: evaluation of literature. Menopause. 2015;22(6):674-684.

147. Cintron D, Lipford M, Larrea-Mantilla L, et al. Efficacy of menopausal hormone therapy on sleep quality: systematic review and meta-analysis. Endocrine. 2017;55(3):702-711.

148. Thomas AJ, Ismail R, Taylor-Swanson L, et al. Effects of isoflavones and amino acid therapies for hot flashes and co-occurring symptoms during the menopausal transition and early postmenopause: a systematic review. Maturitas. 2014;78(4):263-276.

149. Davinelli S, Scapagnini G, Marzatico F, Nobile V, Ferrara N, Corbi G. Influence of equol and resveratrol supplementation on health-related quality of life in menopausal women: a randomized, placebo-controlled study. Maturitas. 2017;96:77-83.

150. De Franciscis P, Grauso F, Luisi A, Schettino MT, Torella M, Colacurci N. Adding Agnus Castus and magnolia to soy isoflavones relieves sleep disturbances besides postmenopausal vasomotor symptoms-long term safety and effectiveness. Nutrients. 2017;9(2):E129.

151. Sateia MJ, Buysse DJ, Krystal AD, Neubauer DN, Heald JL. Clinical practice guideline for the pharmacologic treatment of chronic insomnia in adults: an American Academy of Sleep Medicine Clinical Practice Guideline. J Clin Sleep Med. 2017;13(2):307-349.

152. Trauer JM, Qian MY, Doyle JS, Rajaratnam SM, Cunnington D. Cognitive behavioral therapy for chronic insomnia: a systematic review and meta-analysis. Ann Intern Med. 2015;163(3):191-204.

153. Wu JQ, Appleman ER, Salazar RD, Ong JC. Cognitive behavioral therapy for insomnia comorbid with psychiatric and medical conditions: a meta-analysis. JAMA Intern Med. 2015;175(9): 1461-1472.

154. Sivertsen B, Omvik S, Pallesen S, et al. Cognitive behavioral therapy vs zopiclone for treatment of chronic primary insomnia in older adults: a randomized controlled trial. JAMA. 2006;295(24):2851-2858. 
155. McCurry SM, Guthrie KA, Morin CM, et al. Telephone-based cognitive behavioral therapy for insomnia in perimenopausal and postmenopausal women with vasomotor symptoms: a MsFLASH randomized clinical trial. JAMA Intern Med. 2016;176(7):913-920.

156. Jarrin DC, Chen IY, Ivers H, Morin CM. The role of vulnerability in stress-related insomnia, social support and coping styles on incidence and persistence of insomnia. J Sleep Res. 2014;23(6):681-688.

157. Kline CE, Irish LA, Krafty RT, et al. Consistently high sports/exercise activity is associated with better sleep quality, continuity and depth in midlife women: the SWAN sleep study. Sleep. 2013;36(9): 1279-1288.

158. de Zambotti M, Colrain IM, Sassoon SA, Nicholas CL, Trinder J, Baker FC. Vagal withdrawal during hot flashes occurring in undisturbed sleep. Menopause. 2013;20(11):1147-1153.

159. Lukacs JL, Chilimigras JL, Cannon JR, Dormire SL, Reame NE. Midlife women's responses to a hospital sleep challenge: aging and menopause effects on sleep architecture. JWomens Health (Larchmt). 2004;13(3):333-340.

160. Kalleinen N, Polo-Kantola P, Himanen SL, et al. Sleep and the menopause - do postmenopausal women experience worse sleep than premenopausal women? Menopause Int. 2008;14(3):97-104.

161. Hachul H, Andersen ML, Bittencourt LR, Santos-Silva R, Conway $\mathrm{SG}$, Tufik S. Does the reproductive cycle influence sleep patterns in women with sleep complaints? Climacteric. 2010;13(6):594-603.

162. Hachul H, Frange C, Bezerra AG, et al. The effect of menopause on objective sleep parameters: data from an epidemiologic study in Sao Paulo, Brazil. Maturitas. 2015;80(2):170-178.

163. Morin CM, Espie CA. Insomnia: A Clinical Guide to Assessment and Treatment. New York: Kluwer Academic/Plenum Publishers; 2003.

164. Carney CE, Buysse DJ, Ancoli-Israel S, et al. The consensus sleep diary: standardizing prospective sleep self-monitoring. Sleep. 2012;35(2):287-302.
165. Chung F, Abdullah HR, Liao P. STOP-Bang Questionnaire: a practical approach to screen for obstructive sleep apnea. Chest. 2016;149(3): 631-638.

166. Kline CE, Irish LA, Buysse DJ, et al. Sleep hygiene behaviors among midlife women with insomnia or sleep-disordered breathing: the SWAN sleep study. JWomens Health (Larchmt). 2014;23(11):894-903.

167. North American Menopause Society (NAMS). The 2017 hormone therapy position statement of The North American Menopause Society. Menopause. 2017;24(7):728-753.

168. Ensrud KE, Guthrie KA, Hohensee C, et al. Effects of estradiol and venlafaxine on insomnia symptoms and sleep quality in women with hot flashes. Sleep. 2015;38(1):97-108.

169. Ensrud KE, Joffe H, Guthrie KA, et al. Effect of escitalopram on insomnia symptoms and subjective sleep quality in healthy perimenopausal and postmenopausal women with hot flashes: a randomized controlled trial. Menopause. 2012;19:848-855.

170. Pinkerton JV, Joffe H, Kazempour K, Mekonnen H, Bhaskar S, Lippman J. Low-dose paroxetine $(7.5 \mathrm{mg}$ ) improves sleep in women with vasomotor symptoms associated with menopause. Menopause. 2015;22(1):50-58.

171. Yurcheshen ME, Guttuso T Jr, McDermott M, Holloway RG, Perlis M. Effects of gabapentin on sleep in menopausal women with hot flashes as measured by a Pittsburgh Sleep Quality Index factor scoring model. J Womens Health (Larchmt). 2009;18(9):1355-1360.

172. Joffe H, Guthrie KA, Larson J, et al. Relapse of vasomotor symptoms after discontinuation of the selective serotonin reuptake inhibitor escitalopram results from the menopause strategies: finding lasting answers for symptoms and health research network. Menopause. 2013;20(3):261-268.

173. Bolge SC, Balkrishnan R, Kannan H, Seal B, Drake CL. Burden associated with chronic sleep maintenance insomnia characterized by nighttime awakenings among women with menopausal symptoms Menopause. 2010;17(1):80-6.
Nature and Science of Sleep

\section{Publish your work in this journal}

Nature and Science of Sleep is an international, peer-reviewed, open access journal covering all aspects of sleep science and sleep medicine, including the neurophysiology and functions of sleep, the genetics of sleep, sleep and society, biological rhythms, dreaming, sleep disorders and therapy, and strategies to optimize healthy sleep. The manuscript

\section{Dovepress}

management system is completely online and includes a very quick and fair peer-review system, which is all easy to use. Visit http://www. dovepress.com/testimonials.php to read real quotes from published authors. 